\title{
Radiopharmaceutical therapy in cancer: clinical advances and challenges
}

George Sgouros $\mathbb{1}^{1 凶}{ }^{凶}$, Lisa Bodei $\mathbb{D}^{2}$, Michael R. McDevitt ${ }^{2}$ and Jessie R. Nedrow (1)

Abstract | Radiopharmaceutical therapy (RPT) is emerging as a safe and effective targeted approach to treating many types of cancer. In RPT, radiation is systemically or locally delivered using pharmaceuticals that either bind preferentially to cancer cells or accumulate by physiological mechanisms. Almost all radionuclides used in RPT emit photons that can be imaged, enabling non-invasive visualization of the biodistribution of the therapeutic agent. Compared with almost all other systemic cancer treatment options, RPT has shown efficacy with minimal toxicity. With the recent FDA approval of several RPT agents, the remarkable potential of this treatment is now being recognized. This Review covers the fundamental properties, clinical development and associated challenges of RPT.

Radionuclides

Interchangeable with

'radioactive atoms', 'radioactive elements' and 'radioactive isotopes'. Although these are all correct, 'radionuclide' (not 'radionucleotide') is the preferred term in the context of nuclear medicine, in general, and radiopharmaceutical therapy, in particular.

$\beta$-Particles

Light, energetic electrons that are either positively or negatively charged and emitted spontaneously from atomic nuclei during a nuclear transformation of many radionuclides. These particles are characterized by a spectrum of energies and associated ranges, typically characterized as the maximum or end-point energy or range.

'Johns Hopkins University School of Medicine, Baltimore, MD, USA.

${ }^{2}$ Memorial Sloan Kettering Cancer Center, New York, $N Y$, USA.

$\bigotimes_{e-m a i l: \text { gsgouros@jhmi.edu }}$ https://doi.org/10.1038/ s41573-020-0073-9
Radiopharmaceutical therapy (RPT) is defined by the delivery of radioactive atoms to tumour-associated targets. RPT is a novel therapeutic modality for the treatment of cancer, providing several advantages over existing therapeutic approaches. Unlike radiotherapy, the radiation is not administered from outside the body, but instead is delivered systemically or locoregionally, akin to chemotherapy or biologically targeted therapy. The cytotoxic radiation is delivered to cancer cells or to their microenvironment either directly or, more typically, using delivery vehicles that either bind specifically to endogenous targets or accumulate by a wide variety of physiological mechanisms characteristic of neoplasia, enabling a targeted therapeutic approach. Unlike biologic therapy, it is far less dependent on an understanding of signalling pathways and on identifying agents that interrupt the putative cancer phenotype-driving pathway (or pathways). Notably, the clinical trial failure rate of 'targeted' (that is, biologic) cancer therapies is $97 \%$ (REF. ${ }^{1}$ ), which is in part due to the drugs selected for clinical trial investigation targeting the wrong pathway ${ }^{2}$.

Radionuclides with different emission properties primarily $\beta$-particles or highly potent $\alpha$-particles - are used to deliver radiation. In almost all cases, the radionuclides may be visualized by nuclear medicine imaging techniques to assess targeting of the agent, which provides a substantial advantage over existing therapeutic approaches and enables a precision medicine approach to RPT delivery.

Patients with cancer with distant metastases continue to have a grim prognosis despite ongoing efforts with new chemotherapeutics, small-molecule inhibitors, biologics, immune checkpoint inhibitors and various combinations of these; novel therapeutic approaches are therefore vital. Compared with almost all other systemic cancer treatment options, RPT has shown efficacy with minimal toxicity ${ }^{3}$. In addition, unlike chemotherapy, responses with RPT agents typically do not require many months (or cycles) of therapy and are often observed after a single or at most five injections; side effects such as alopecia or peripheral neuropathy are generally less severe than with chemotherapy, if observed at all.

RPT development is a multidisciplinary endeavour, requiring expertise in radiochemistry, radiobiology, oncology, pharmacology, medical physics and radionuclide imaging and dosimetry - most pharmaceutical companies are not familiar with the radiation and radionuclide aspects of RPT and the deployment of RPT agents for cancer therapy is also unfamiliar to the oncology community. It is a therapeutic modality that is not consistently identified with any one group of practitioners and it lacks a constituency. For many decades RPT has been a treatment modality of last resort and available only in small clinical trials or as part of compassionate care from a small number of institutions in Europe and even fewer in the USA and the rest of the world. In the sense that RPT has no well-defined community of stakeholders it has been an 'orphan treatment' modality for many years.

However, the remarkable potential of RPT directed against primary cancers as well as distant metastases, is now being recognized as an effective, safe and economically and logistically viable treatment modality, receiving renewed attention from both small and large pharmaceutical companies ${ }^{4}$. The recent approval of 


\section{$\alpha$-Particles} particles ejected spontaneously from the nucleus of some radionuclides that are identical to a helium nucleus (mass number of 4 and electrical charge of +2 ). The DNA damage resulting from these short-range particles per unit of energy deposition is greater and more complex than that associated with $\beta$-particles.

Absorbed dose

The energy absorbed per unit mass of tissue.

Gy

Abbreviation for the SI unit (gray) for radiation dose $\left(1 \mathrm{~Gy}=1 \mathrm{~J} \mathrm{~kg}^{-1}\right)$. It is the absorbed dose per unit mass of tissue. The corresponding (100 rad = $1 \mathrm{~Gy}$ ). The rad is not recommended for use in the scientific literature.
Positively charged, heavy CGS unit is the rad

$\beta$-particle-emitting RPT agents that act against neuroendocrine cancers and phaeochromocytomas, the approval of an $\alpha$-emitter RPT for bone metastases of prostate cancer and the highly promising clinical and preclinical preliminary results with RPT agents using other $a$-particle-emitting radionuclides has reignited interest in RPT.

This Review provides an overview of the radiochemistry and physics aspects needed to understand the fundamentals of RPT. The different categories of RPT agents in use and in the clinic for the treatment of cancer and the challenges associated with their development and application are discussed. Notably, many other RPT agents are in preclinical development, but their discussion is beyond the scope of this Review. Similarly, while RPT also has applications in non-oncological disorders, such as rheumatoid arthritis and polyarthritis, these will not be discussed here, and the reader is referred to recent reviews on these topics ${ }^{5,6}$.

\section{Overview of RPT}

\section{Mechanism and biological effects}

The mechanism of action for RPT is radiation-induced killing of cells. Investigation into the effects of radiation
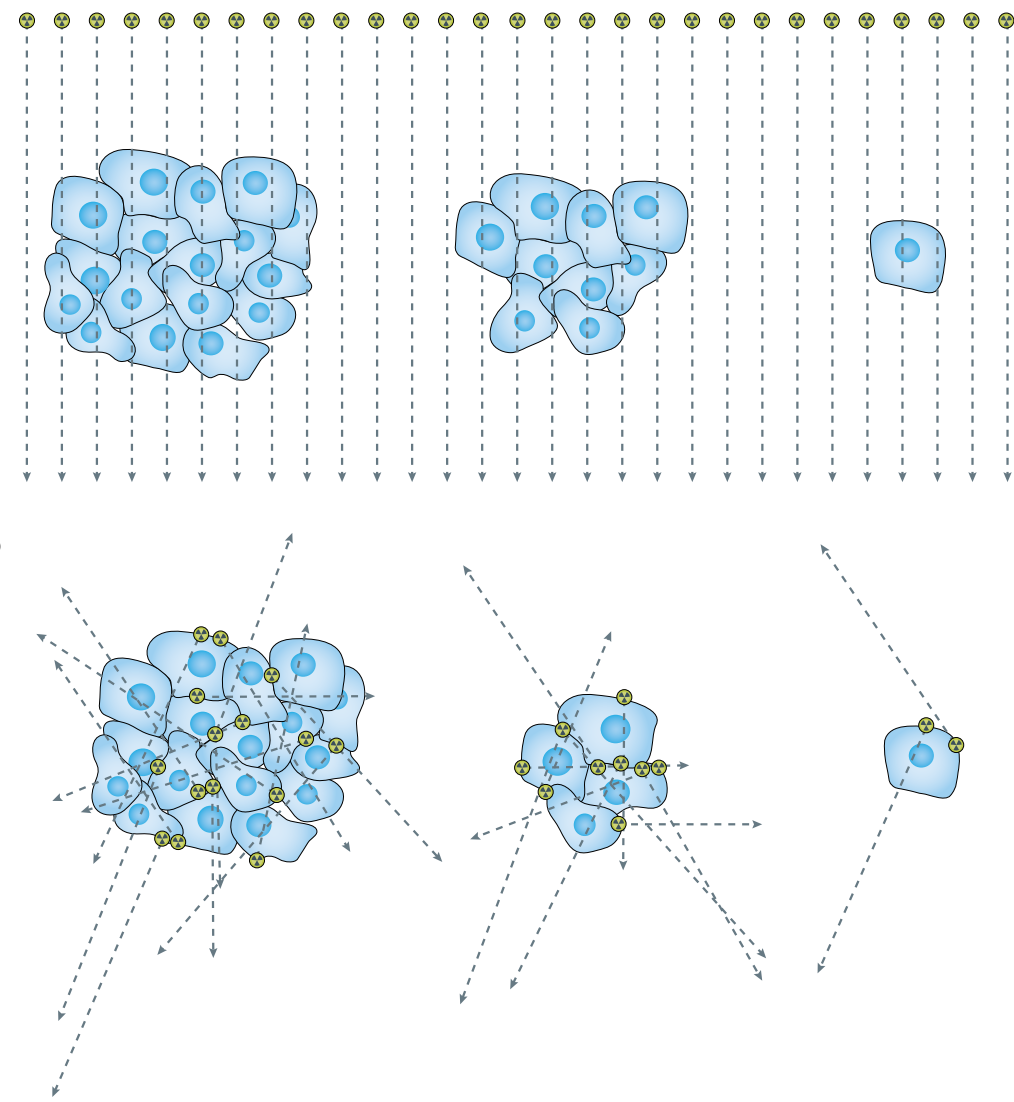

Fig. 1 | Tumour cell irradiation: radiotherapy versus radiopharmaceutical therapy. a $\mid$ An external beam delivers the same absorbed dose per cell regardless of the number of cells. $\mathbf{b} \mid$ In radiopharmaceutical therapy, the absorbed dose delivered per cell by emissions originating from cells is influenced by the range of the emissions, the number of cells that are clustered together and the number of cells that have been targeted. A single cell is very difficult to sterilize with radiopharmaceutical therapy. If the range of the emitted particle is much longer than the dimension of the cell nucleus, a smaller fraction of the total emitted energy will be absorbed in the nucleus. on tissues and tumours began soon after the discovery of radiation and radioactivity. RPT has the benefit of drawing on the substantial knowledge base of radiotherapy ${ }^{7}$. However, RPT differs from radiotherapy, and it is important to understand how those elements unique to RPT influence therapy.

The essential questions for RPT are where does the agent localize and for how long? As noted in the section entitled 'Dosimetry', answers to these questions inform the tumour versus normal tissue absorbed dose and provide a measure of potential treatment success.

The biological effects of a given absorbed dose for a tumour depend on the rate at which the dose is delivered $^{8-10}$. A dose of 30 Gy delivered to a tumour over a period of many weeks at a dose rate that is exponentially decreasing, as is typically the case with RPT, will have a very different effect from that of the same amount delivered at the much higher dose rates used in radiotherapy (for example, daily, 2-Gy fractions over 15 days). The difference in biological outcome will depend on the biological repair and radiosensitivity properties of the tumour. Dose-rate considerations also apply to normal organs ${ }^{11-13}$.

Another fundamental distinguishing feature important for understanding this treatment modality is the diminishing curative potential with reduced target cell number (FIG. 1). In radiotherapy the probability of killing all cells for a given absorbed dose increases as the number of target cells decreases - fewer cells to kill for a given radiation absorbed dose increases the chance that all of the cells will be killed. By contrast, fewer cells do not translate into a greater tumour control probability in RPT. This is because the radiation is not delivered uniformly to all cells. If the emitted radiation originates from a radionuclide on the surface of tumour cells, fewer cells leads to a smaller fraction of the emitted energy being deposited into the targeted cells ${ }^{14}$. This is balanced, in part, by the greater concentration that may be achieved in smaller clusters of cells relative to large measurable tumours.

\section{Radionuclides used for RPT}

One of the hallmarks of RPT is its ability to deliver highly potent forms of radiation directly to tumour cells. Three different types of radiation are relevant to understanding RPT: photons, electrons and $\alpha$-particles ${ }^{15}$.

Photons come in two 'flavours' $-X$-rays and $\gamma$-rays. The former are derived from orbital electron transitions and are typically lower in energy than $\gamma$-rays. Radionuclide photon emissions are useful for imaging the distribution of the RPT but not for localized delivery of cytotoxic radiation. Although a wide range of photon energies may be imaged (70-400 keV), photon emission energies in the range from 100 to $200 \mathrm{keV}$ are optimal for all nuclear medicine imaging cameras ( $\gamma$-cameras and single-photon emission computed tomography (SPECT) cameras). A number of radionuclides also emit positrons which lead to the emission of $511-\mathrm{keV}$ photons that are detected by positron emission tomography (PET) cameras.

Electron emissions are classified by energy and also by the type of decay. Auger electrons, $\beta$-particles and 

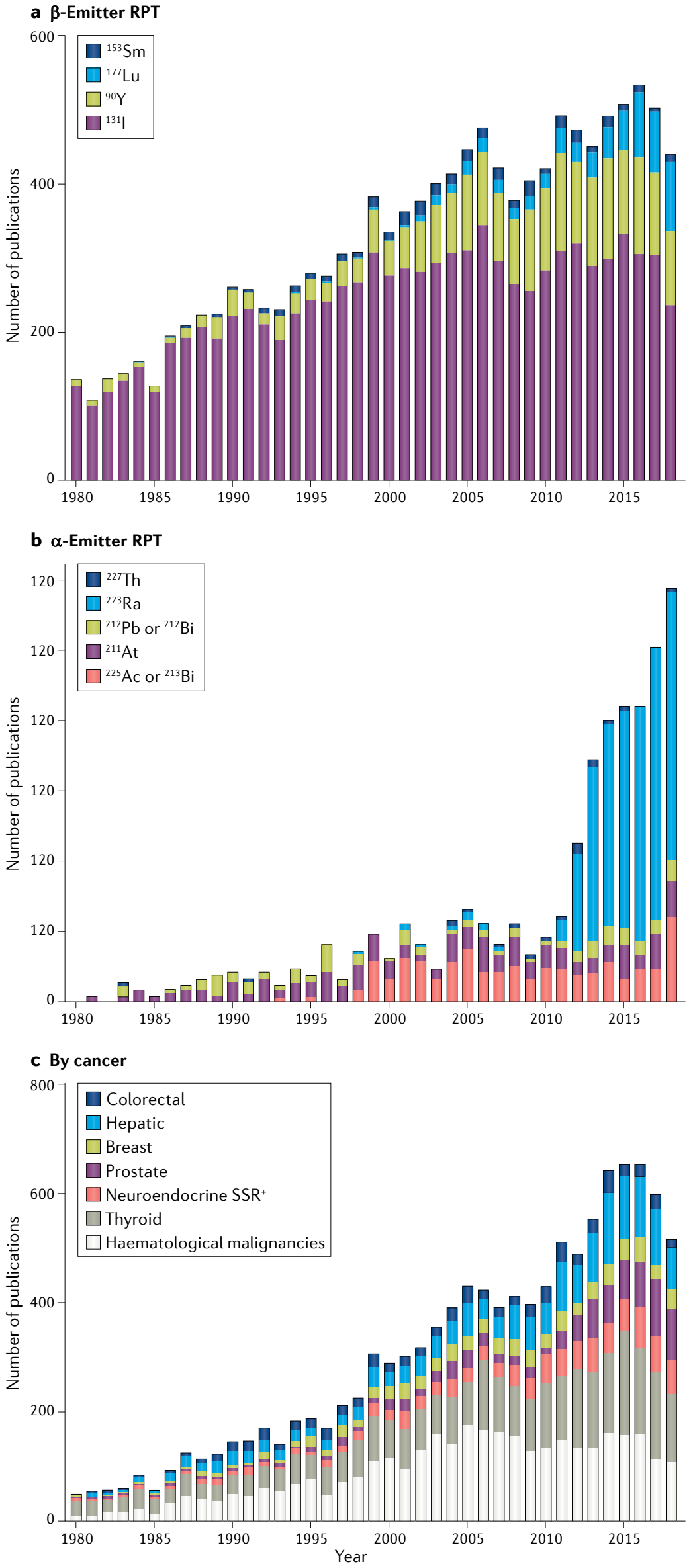

monoenergetic electrons are relevant to RPT. Auger electrons are generated from suborbital transitions. They are typically very-short-range emissions, of the order of 1-1000 nm, depending on their emission energy. If the RPT drug localizes within the cell nucleus, these emissions could be highly cytotoxic ${ }^{13,16-20}$. Auger electron-emitter RPT has not been widely adopted, however. Although preclinical studies have shown substantial therapeutic efficacy, the small number of human investigations did not lead to clinical efficacy ${ }^{21-24}$. Human studies using locoregional administration showed promise in terms of tumour cell incorporation of the Auger emitters ${ }^{17,20,25}$. The requirement that these agents be incorporated into the DNA and also their unfavourable pharmacokinetics are thought to be the reasons underlying the lack of efficacy. Encouraged by ongoing technological developments that could overcome the factors listed above, these agents continue to be of interest to the RPT community ${ }^{26}$.

$\beta$-Particles are electrons emitted from the nucleus. They typically have a longer range in tissue (of the order of $1-5 \mathrm{~mm}$ ) and are the most frequently used emission type for RPT agents. This is in part because $\beta$-particle-emitting radionuclides are widely available, many of which also emit photons in an energy range that is easily imaged. The $\beta$-particle emitters samarium-153, lutetium-177, yttrium-90 and I-131 have been introduced and commonly used over the last 40 years (FIG. 2a; TABLES 1,2). The most familiar and frequently used of these is iodine-131, which is used to treat thyroid cancer. A number of other $\beta$-emitting radionuclides have been investigated or considered ${ }^{27-31}$ but for several reasons including lack of availability, difficult radiochemistry or perhaps, most prominently, the absence of a commercial justification to overcome the regulatory and financial hurdles involved in their clinical translation - these agents have not been widely adopted ${ }^{32}$.

A variety of reasons for the progression and shift to different radionuclides may be invoked to explain the changes and additions of the different $\beta$-particle emitters used over time. For example, in an early theoretical evaluation of different radionuclides, yttrium-90 ranked second to rhenium-186 in a list of nine radionuclides considered ${ }^{12}$. In that ranking, the main criterion was the tumour to non-tumour-absorbed dose ratio. This was a theoretical calculation obtained for different radionuclides using typical radiolabelled antibody pharmacokinetics for tumour targeting and

Fig. 2 | Publications per year related to RPT. a | Number of publications related to radiopharmaceutical therapy (RPT) listed in the PubMed database per indicated year for each of the indicated $\beta$-particle-emitting radionuclides. The figure shows the introduction and expanded use of new $\beta$-particleemitting radionuclides for RPT. For example, lutetium-177 was first described for use in RPT in 1991 (REF. ${ }^{301}$ ). By 2018 the number of publications related to lutetium-177 and RPT was about the same as that for yttrium-90. b| Publications per year for RPT with different $\alpha$-emitting radionuclides. c| Number of publications related to RPT listed in the PubMed database per indicated year for each of the indicated malignancies. See Supplementary information for search terms used. SSR, somatostatin receptor. 
Table 1 | Radionuclide properties

\begin{tabular}{|llll|}
\hline Radionuclide & $\begin{array}{l}\text { Therapeutic } \\
\text { emission }\end{array}$ & $\begin{array}{l}\text { Approximate } \\
\text { emission range in } \\
\text { tissue (mm) }\end{array}$ & $\begin{array}{l}\text { Radionuclide } \\
\text { half-life }\end{array}$ \\
\hline Yttrium-90 & $\beta^{-}$ & 5.30 & 64.1 hours \\
\hline lodine-131 & $\beta^{-}$ & 0.8 & 8.0 days \\
\hline Samarium-153 & $\beta^{-}$ & 0.4 & 46.5 hours \\
\hline Lutetium-177 & $\beta^{-}$ & 0.62 & 6.6 days \\
\hline Astatine-211 & $\alpha$ & 0.05 & 7.2 hours \\
\hline Lead-212/bismuth-212 & $\beta^{-} / \alpha$ & $<0.1 / 0.05$ & 10.6 hours/1.0 hours \\
\hline Radium-223 & $\alpha$ & $0.05-0.08$ & 11.4 days \\
\hline Actinium-225 & $\alpha$ & $0.05-0.08$ & 10.0 days \\
\hline Thorium-227 & $\alpha$ & $0.05-0.08$ & 18.7 days \\
\hline
\end{tabular}

Half-lives from REF. ${ }^{297}$, ranges from REFS ${ }^{298-300}$.

\section{Photons}

A photon is a quantum of highfrequency electromagnetic

radiation that is emitted spontaneously, either during a nuclear transformation ( $\gamma$-rays) or as a result of orbital electron transitions (X-rays). In the context of radiation delivery, the energy or frequency of photons is sufficient to ionize atoms and lead to potential DNA damage.

X-rays

Photons emitted as a result of orbital electron transitions.

$\gamma$-Rays

Photons emitted during a nuclear transformation of a radionuclide.

keV

The abbreviation for a unit of energy (kiloelectronvolts) typically used to represent the energy associated with radionuclide emissions.

Auger electrons Electrons ejected during suborbital transitions of an atom, typically following spontaneous nuclear transitions (radioactive decay).

Activities

A measure of radioactivity, typically in the unit of becquerel $(\mathrm{Bq})$ or millicurie (mCi; the CGS unit). Radioactivity corresponds to the number of radionuclide transformations per unit time.

Theranostic

The general concept of using a radionuclide-labelled agent that may be imaged to guide radiopharmaceutical therapy a radionuclide that may be used for both imaging and therapy. organ clearance. This optimum reflected the 64.2-hour half-life of yttrium-90 and its high-energy $\beta$-particle, which was deemed favourable for uniformly irradiating tumours. Like iodine-131, the adoption of yttrium-90 for RPT is likely based on its history and widespread availability. In the 1970s it was used in colloidal form, primarily to treat rheumatoid conditions ${ }^{33,34}$. Efforts to conjugate yttrium-90, a radiometal, were unsuccessful ${ }^{35}$ until a radiometal conjugation chemistry that retained stability in vivo was developed ${ }^{36,37}$. Clinical trials using yttrium-90-labelled antibodies as RPT agents initially focused on ovarian cancer ${ }^{38,39}$ and subsequently on haematological cancers ${ }^{40-42}$, as well as radiopeptide therapy ${ }^{43}$. Yttrium-90 continues to be a popular radionuclide for RPT because of the clinical impact of yttrium-90-impregnated microspheres that are used for treatment of hepatic metastases ${ }^{44-48}$. Although yttrium- 90 has been imaged, imaging generally requires high activities (more than $300 \mathrm{MBq})^{49}$. Such activities are typically achieved only in microsphere therapies. Lutetium-177 gained popularity because it emits photons in the 100-200-keV optimal imaging range and has a $\beta$-particle energy appropriate for therapy. These factors, along with a half-life that is compatible with the pharmacokinetics of both antibodies and peptides, make this radionuclide a theranostic in that the same radionuclide may be used to assess tumour uptake and the extent of cancer, and also as a treatment ${ }^{50}$. It is produced in a reactor and is therefore widely available ${ }^{51}$, with a relatively straightforward conjugation chemistry ${ }^{52}$.

a-Particles are helium nuclei (two protons and two neutrons) that are emitted from the nucleus of a radioactive atom. Depending on their emission energy, they can travel $50-100 \mu \mathrm{m}$ in tissue. They are positively charged and are orders of magnitude larger than electrons. The amount of energy deposited per path length travelled (designated 'linear energy transfer') of $\alpha$-particles is approximately 400 times greater than that of electrons. This leads to substantially more damage along their path than that caused by electrons. An a-particle track leads to a preponderance of complex and largely irreparable DNA double-strand breaks ${ }^{53,54}$. The absorbed dose required to achieve cytotoxicity relates to the number of $\alpha$-particles traversing the cell nucleus. With use of this as a measure, cytotoxicity may be achieved with a range of 1 to $20 \alpha$-particle traversals of the cell nucleus ${ }^{53}$. The resulting high potency, combined with the short range of $\alpha$-particles (which reduces normal organ toxicity), has led to substantial commercial interest in developing $\alpha$-particle-emitting RPT agents ${ }^{55}$. The a-particle emitters introduced and used for RPT over the last 40 years include astatine-211, bismuth-212, lead-212, bismuth-213, actinium-225, radium-223 and thorium-227 (FIG. 2b; TABLES 1,2).

The a-particle-emitting RPT agent radium-223 dichloride (Xofigo) was approved by the FDA in May 2013. At the time it was approved, effective therapies for patients with castration-resistant prostate cancer that had metastasized to bone did not exist. The very encouraging clinical trial results ${ }^{56}$ and the subsequent FDA approval were instrumental in the renewed interest in RPT, in general, and $\alpha$-emitter RPT, in particular. Subsequently, individual-patient demonstrations of $a$-emitter potency have reinforced the interest in a-particle-emitting RPT ${ }^{57,58}$. Overall, there has been an almost sixfold increase in the number of studies related to $\alpha$-emitters for RPT over the past 10 years. Ongoing investigations of radium-223 in combination with other therapeutics ${ }^{59}$ that act against other bone-seeking cancers $^{60-62}$ have led to the recent high prevalence of radium-223 RPT studies.

There are more than 300 radionuclides that are potentially relevant to medicine and biology ${ }^{63}$. TABLE 1 details the subset of these that are RPT agents of current interest, along with properties relevant to their use in RPT.

\section{Dosimetry}

Response and toxicity prediction is essential for the rational implementation of cancer therapy. The biological effects of radionuclide therapy are mediated by a well-defined physical quantity, the absorbed dose $(D)$, which is defined as the energy absorbed per unit mass of tissue. The long and well-established cancer treatment experience in radiotherapy has provided ample evidence that absorbed dose may be used to predict biological response ${ }^{64}$. In chemotherapy, targeted biologic therapy and immunotherapy, there is no dosimetry analogue. Dosimetry as implemented in RPT may be thought of as the ability to perform the equivalent of a pharmacodynamic study in treated patients in real time. Dosimetry analysis may be performed as part of patient treatment to calculate tumour versus normal organ absorbed dose and therefore the likelihood of treatment success. The ability to rapidly assay genetic and epigenetic characteristics of tumour samples comes closest to providing the kind of information that RPT dosimetry provides regarding the potential efficacy and toxicity of a therapeutic agent in an individual patient.

The mathematical formalism and tools available to perform dosimetry have evolved over time. The initial impetus for estimating absorbed dose was to assess the potential risks of nuclear medicine diagnostic imaging agents. Dosimetry calculations intended to assess risk are performed for an anatomical geometry designed to represent the average patient population rather than 
Table 2 | Selected RPT agents that are on the market or under development

\begin{tabular}{|c|c|c|c|c|c|c|}
\hline RPT agent & Company & Indication & Properties & Development phase & NCT number & Refs \\
\hline $\begin{array}{l}\text { Radium-223 } \\
\text { chloride }^{\mathrm{a}}\end{array}$ & Bayer & Bone metastasis & Calcium analogue & $\begin{array}{l}\text { Commercially } \\
\text { available }\end{array}$ & - & $56,127-131$ \\
\hline $\begin{array}{l}{ }^{90} \text { Y-loaded glass } \\
\text { microspheres }\end{array}$ & BTG & $\begin{array}{l}\text { Hepatic } \\
\text { malignancies }\end{array}$ & $\begin{array}{l}\text { Radioembolization of liver } \\
\text { microvasculature }\end{array}$ & $\begin{array}{l}\text { Commercially } \\
\text { available }\end{array}$ & - & $274-279$ \\
\hline $\begin{array}{l}{ }^{90} \text { Y-loaded resin } \\
\text { microspheres }\end{array}$ & $\begin{array}{l}\text { CDH Genetech/ } \\
\text { Sirtex }\end{array}$ & $\begin{array}{l}\text { Hepatic } \\
\text { malignancies }\end{array}$ & $\begin{array}{l}\text { Radioembolization of liver } \\
\text { microvasculature }\end{array}$ & $\begin{array}{l}\text { Commercially } \\
\text { available }\end{array}$ & - & 274-279 \\
\hline${ }^{153}[\mathrm{Sm}]$ lexidronam & Lantheus & Cancer bone pain & $\begin{array}{l}\text { Binding to hydroxyapatite } \\
\text { matrix }\end{array}$ & $\begin{array}{l}\text { Commercially } \\
\text { available }\end{array}$ & - & $132-134$ \\
\hline $\begin{array}{l}{ }^{177} \text { Lu-labelled } \\
\text { DOTATATE }\end{array}$ & Novartis/AAA & $\begin{array}{l}\text { Neuroendocrine } \\
\text { tumours }\end{array}$ & SSR-mediated binding & $\begin{array}{l}\text { Commercially } \\
\text { available }\end{array}$ & - & $192,194-196,200$ \\
\hline${ }^{131}$-labelled aCD45 & $\begin{array}{l}\text { Actinium } \\
\text { Pharmaceuticals }\end{array}$ & $\begin{array}{l}\text { Bone marrow } \\
\text { transplant } \\
\text { preparation }\end{array}$ & $\begin{array}{l}{ }^{131} \mid \text {-based antibody targeting } \\
C D 45^{+} \text {cells for bone } \\
\text { marrow ablation before } \\
\text { transplantation }\end{array}$ & Phase III; recruiting & NCT02665065 & 229-231 \\
\hline $\begin{array}{l}{ }^{177} \text { Lu-labelled } \\
\text { PSMA-617 }\end{array}$ & $\begin{array}{l}\text { Novartis/ } \\
\text { Endocyte }\end{array}$ & $\begin{array}{l}\text { Prostate } \\
\text { cancer, tumour } \\
\text { neovasculature }\end{array}$ & PSMA-mediated binding & $\begin{array}{l}\text { Phase III; active, not } \\
\text { recruiting }\end{array}$ & NCT03511664 & $166-169$ \\
\hline \multirow{2}{*}{$\begin{array}{l}{ }^{177} \text { Lu-labelled } \\
\text { NeoBOMB1 }\end{array}$} & \multirow[t]{2}{*}{ Novartis/AAA } & \multirow[t]{2}{*}{$\mathrm{GRPR}^{+}$tumours } & \multirow[t]{2}{*}{ GRPR binding } & Phase II; completed & NCT03724253 & \multirow[t]{2}{*}{$202-210$} \\
\hline & & & & Phase I/II; completed & NCT02931929 & \\
\hline${ }^{166} \mathrm{Ho}$ microspheres & Terumo & $\begin{array}{l}\text { Hepatic } \\
\text { malignancies }\end{array}$ & $\begin{array}{l}\text { Radioembolization of liver } \\
\text { microvasculature }\end{array}$ & $\begin{array}{l}\text { Phase Il; unknown } \\
\text { recruitment status }\end{array}$ & NCT02067988 & $280-282$ \\
\hline $\begin{array}{l}{ }^{225} \mathrm{Ac} \text {-labelled } \\
\mathrm{aCD}^{2} 3^{\mathrm{a}}\end{array}$ & $\begin{array}{l}\text { Actinium } \\
\text { Pharmaceuticals }\end{array}$ & Leukaemia, MDS & CD33 antibody $\alpha$-targeting & Phase I; withdrawn & NCT03705858 & $239,241-243$ \\
\hline $\begin{array}{l}\text { 227Th-labelled } \\
\text { MSLN-TTC }\end{array}$ & Bayer & Mesothelin ${ }^{+}$tumours & $\begin{array}{l}\text { Anti-mesothelin- } \alpha \text {-emitter } \\
\text { immunoconjugate }\end{array}$ & Phase I; recruiting & NCT03507452 & 248,249 \\
\hline $\begin{array}{l}\text { 227Th-labelled } \\
\text { PSMA-TTC }\end{array}$ & Bayer & $\begin{array}{l}\text { Prostate, tumour } \\
\text { neovasculature }\end{array}$ & $\begin{array}{l}\text { PSMA-targeting a-emitter } \\
\text { immunoconjugate; } \text { PSMA }^{+} \\
\text {prostate cancer targeting }\end{array}$ & Phase I; recruiting & NCT03724747 & 250,251 \\
\hline $\begin{array}{l}\text { 227Th-labelled } \\
\text { aCD22-TTC }\end{array}$ & Bayer & Lymphoma & $\begin{array}{l}\text { Anti-CD22- } \alpha \text {-emitter } \\
\text { immunoconjugate; CD22+ } \\
\text { tumours (lymphoma) }\end{array}$ & $\begin{array}{l}\text { Phase I; active, not } \\
\text { recruiting }\end{array}$ & NCT02581878 & 252 \\
\hline $\begin{array}{l}{ }^{177} \text { Lu-labelled } \\
\text { СТT-1403 }\end{array}$ & $\begin{array}{l}\text { Cancer Targeted } \\
\text { Technologies }\end{array}$ & $\begin{array}{l}\text { Prostate, tumour } \\
\text { neovasculature }\end{array}$ & PSMA-mediated binding & $\begin{array}{l}\text { Phase I; active, not } \\
\text { recruiting }\end{array}$ & NCT03822871 & $65,184-191$ \\
\hline \multirow{4}{*}{$\begin{array}{l}\text { 131|-labelled } \\
\text { CLR } 131\end{array}$} & \multirow[t]{4}{*}{ Cellectar } & \multirow{4}{*}{$\begin{array}{l}\text { Paediatric cancer, } \\
\text { head and neck } \\
\text { cancer, multiple } \\
\text { myeloma, leukaemia, } \\
\text { lymphoma }\end{array}$} & \multirow{4}{*}{$\begin{array}{l}{ }^{131} \text {-labelled phospholipid } \\
\text { ether analogue targeting } \\
\text { cancer cell-specific lipid raft } \\
\text { microdomains }\end{array}$} & Phase I; recruiting & NCT03478462 & \multirow[t]{4}{*}{ 65,184-191 } \\
\hline & & & & Phase I; suspended & NCT04105543 & \\
\hline & & & & lowing to & NCT02952508 & \\
\hline & & & & $\mathrm{Ph}$ & & \\
\hline \multirow{2}{*}{$\begin{array}{l}\text { 131-labelled } \\
\text { CLR1404 }\end{array}$} & \multirow[t]{2}{*}{ Cellectar } & \multirow{2}{*}{$\begin{array}{l}\text { Unresponsive solid } \\
\text { tumour, multiple } \\
\text { myeloma }\end{array}$} & \multirow{2}{*}{$\begin{array}{l}{ }^{131} \text {-labelled phospholipid } \\
\text { ether analogue targeting } \\
\text { cancer cell-specific lipid raft } \\
\text { microdomains }\end{array}$} & Phase I; not recruiting & NCT02278315 & \multirow[t]{2}{*}{ 65,184-191 } \\
\hline & & & & Phase I; completed & NCT01495663 & \\
\hline $\begin{array}{l}{ }^{225} \mathrm{Ac} \text {-labelled } \\
\text { FPX- } 01^{\mathrm{a}}\end{array}$ & JEJ/Fusion Pharma & $\begin{array}{l}\text { NSCLC, pan-cancer } \\
\text { target }\end{array}$ & $\begin{array}{l}\text { Insulin growth factor } 1^{+} \\
\text {tumours }\end{array}$ & Phase I; recruiting & NCT03746431 & 246 \\
\hline
\end{tabular}


Table 2 (cont.) | Selected RPT agents that are on the market or under development

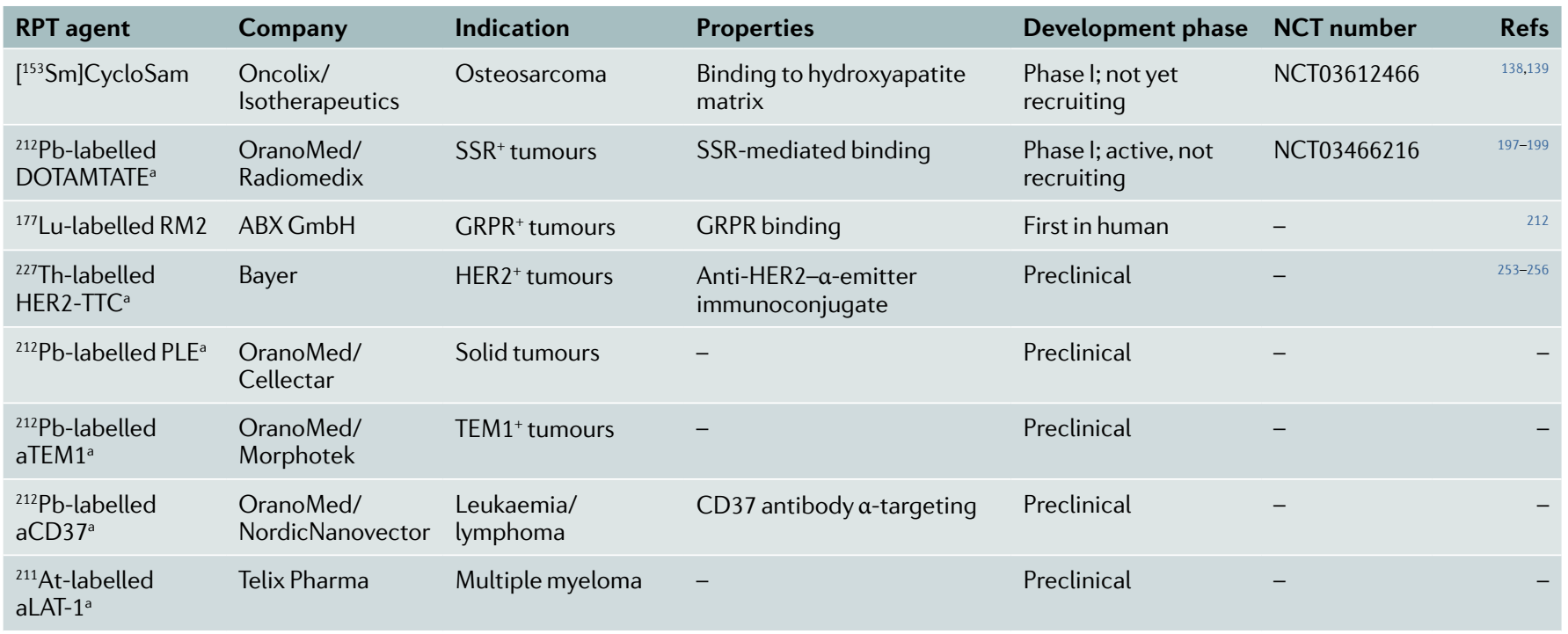

The list is not exhaustive and includes only agents that are being developed by a commercial sponsor. ${ }^{a} \alpha$-Emitter-based radiopharmaceutical therapy (RPT) agents. MDS, myelodysplastic syndrome; mIBG, meta-iodobenzylguanidine; NSCLC, non-small-cell lung cancer; PSMA, prostate-specific membrane antigen; SSR, somatostatin receptor.

Time-versus-activity curves The amount of radioactivity in a particular region of the body as a function of time. Time-versus-activity curves are used in absorbed dose calculations; they may be obtained from imaging or direct sampling (for example, urine or serial biopsy or from animal studies). any one particular patient. The scheme used to calculate these values is shown in BOX 1. This scheme provides the foundation for RPT dosimetry but because the calculation is oriented towards assessing radiation-induced risks of diagnostic imaging, it is not appropriate for toxicity and antitumour efficacy evaluations relevant to RPT. The dosimetry methods summarized in BOX 2 describe a scheme appropriate to RPT. The implications of adopting such a scheme and necessary extensions, not summarized in BOX 2, are briefly described below.

Image-based, patient-specific dosimetry allows the distribution of the agent in tumours and normal organs to be quantified ${ }^{65}$. The amount of RPT agent that concentrates in the tumour can be increased by increasing the administered activity, which also impacts the tumour-absorbed dose. Dosimetry analysis following a low-activity administration has the potential to inform the amount of activity to administer for subsequent therapy. For example, from radiotherapy experience, an absorbed dose of 60-70 Gy is required to achieve greater than 3-year tumour control for patients with osteosarcoma ${ }^{66,67}$. Although such high average tumour-absorbed doses can be achieved with high administered activities, studies have indicated that intratumour variability can be large, with some portions of a tumour meeting and exceeding this dose range, but the overall average being well below therapeutic efficacy ${ }^{68}$. These observations led to a rational, absorbed-dose driven approach for combining radiotherapy with $\mathrm{RPT}^{69}$

In the context of RPT, organ toxicity is usually reflected not by a whole-organ absorbed dose but rather by absorbed dose 'hot spots'. This is particularly so if such regions of high absorbed dose correspond to organ subregions that are critical to organ function. For example, some RPT agents (primarily peptides) concentrate and are retained in the renal cortex, so the absorbed dose in the renal cortex better predicts toxicity than the absorbed dose in the whole kidney volume ${ }^{70}$. For certain
RPT agents, the biologically relevant region can be microscopic (for example, the kidney nephron ${ }^{71}$ or the collecting ducts of the salivary glands ${ }^{72,73}$ ). The converse is also true. Estimates of the average absorbed dose in bone marrow do not predict the very low haematological toxicity of radium-223 dichloride (lower than that of almost all other RPT agents). In a pivotal randomized clinical trial, the grade 3 or grade 4 haematological toxic effects observed in patients receiving radium-223 dichloride (Xofigo) included neutropenia (2\%), thrombocytopenia $(3 \%)$, leukopenia $(3 \%)$ and pancytopenia $(1 \%)^{56}$. This discrepancy could be resolved by a calculation that considered the microscale distribution of radium-223 in the bone marrow. Such an analysis demonstrated that because of the short range of $\alpha$-particle emissions and the known localization of the RPT agent on the trabecular bone surface, only haematopoietic (bone marrow) cells within $80 \mu \mathrm{m}$ of the bone surface were irradiated, meaning that most of the bone marrow space was not irradiated and, accordingly, the average bone marrow absorbed dose was not predictive of toxicity ${ }^{74}$.

Current imaging techniques do not possess the resolution required to resolve activity distributions at the microscopic scale. However, by pairing whole-organ macroscale measurements that can be performed in humans with microscale information that can be obtained from preclinical studies, it is possible to extract microscale information from macroscale measurements. A contour may be drawn on an image obtained with a patient imaging modality such as PET/CT or SPECT/CT that encompasses the entire organ (for example, kidney) or macroscopic subcompartments within the organ (for example, renal cortex). These macroscale contours may be used to obtain time-versus-activity curves (TACs) for the entire organ or macroscale subcompartments within the organ. Microscale structures within these macroscale contours may be obtained in preclinical models by tissue extraction and high-resolution imaging of tissue 
Box 1 | Scheme appropriate for dosimetry of imaging agents and risk evaluation

One of the earliest advances in establishing a dosimetry formalism for risk evaluation applicable to radionuclides was the reformulation of the fundamental definition (Eq. 1) of absorbed dose, $D$, given by the differential of the average energy imparted to matter $(\bar{\varepsilon})$ divided by the mass $(m)$ that has absorbed $\bar{\varepsilon}$ :

$$
D=\frac{\mathrm{d} \bar{\varepsilon}}{\mathrm{d} m} .
$$

Equation 1 was reformulated by the Medical Internal Radiation Dose Committee into a product of two quantities, one that depends on the pharmacokinetics of the radionuclide in the body and one that is a property of the radionuclide and the representative patient anatomy used for the calculation ${ }^{81,306}$ :

$$
D\left(r_{\mathrm{T}} \leftarrow r_{\mathrm{S}}\right)=\widetilde{A}\left(r_{\mathrm{S}}\right) S\left(r_{\mathrm{T}} \leftarrow r_{\mathrm{S}}\right) .
$$

Equation 2 gives the absorbed dose, $D$, for a target region, $r_{\mathrm{T}}$, from the total number of radionuclide transformations or decays, $\widetilde{A}\left(r_{S}\right)$, that have occurred in the source region, $r_{\mathrm{s}} . \widetilde{A}\left(r_{\mathrm{S}}\right)$ is calculated with Eq. 3. It is obtained by integrating the radioactivity in $r_{\mathrm{s}}$ (denoted by $\left.A\left(r_{s}, t\right)\right)$ from the time of injection, at $t=0$ to a time that is long enough so that all of the radioactivity in a particular tissue has either cleared from the tissue or decayed (in almost all diagnostic nuclear medicine and radiopharmaceutical therapy scenarios this is set to infinity):

$$
\widetilde{A}\left(r_{S}\right)=\int_{0}^{\infty} A\left(r_{S}, t\right) d t .
$$

Part a of the figure illustrates the different elements involved in calculating the timeintegrated activity for a given source region. The circles correspond to measurements of the radioactivity (for example, by imaging) in the chosen source region at different points in time. The line going through the circles corresponds to a fit to an equation that best matches the measured data. The exponential equation shown in the figure is typically used for tissues that rapidly accumulate some fraction of the administered activity and then clear it over time. $A\left(r_{s}, t\right)$ can be equated to an exponential function representing clearance of the radioactivity from a particular source tissue with a clearance rate that is the sum of the biological clearance rate $\left(\lambda_{B}\right)$ and the physical decay rate $\left(\lambda_{p}\right) . \lambda_{B}+\lambda_{p}=\lambda_{e}$, the effective (e) clearance rate; $A_{0}$ and $f_{\mathrm{s}}$ are the administered activity and fraction in the source tissue (s), respectively, at $\mathrm{t}=0$ (i.e., back extrapolated from the fit to the $y$ axis). The area under this time-versus-activity curve is the total number of disintegrations, formally known as the time-integrated activity (TIA).

Detailed descriptions of how these measurements should be made using quantitative imaging methods have been published ${ }^{80,83,307}$.

The second quantity, the $S$ value, provides the energy absorbed in the target tissue per unit disintegration in the source tissue. Part b of the figure shows Eq. 2 with the individual terms making up the $S$ value explicitly identified. The $S$ value incorporates quantities reflecting the total emitted energy per radionuclide disintegration, $\Delta$, the fraction of the energy emitted from a source region $\left(r_{S}\right)$ that is absorbed in a target region $\left(r_{T}\right), \phi\left(r_{T} \leqslant r_{S}\right)$, and the mass of the target, $M\left(r_{s}\right)$. Further mathematical details regarding this quantity and $S$ value-based dosimetry, in general, are available in $\mathrm{REF}^{81}$.
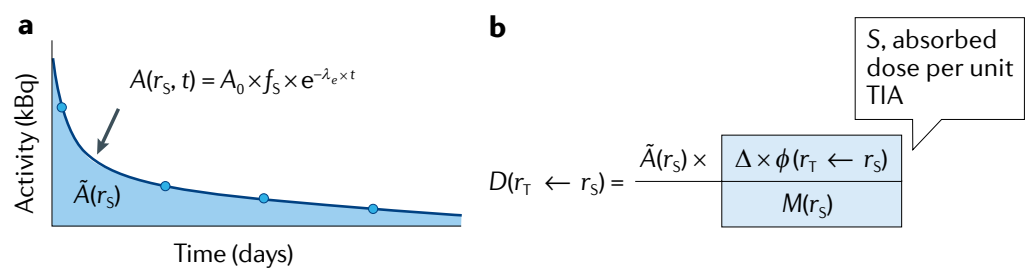

sections, for example $\mathrm{e}^{75-77}$. These data may be used to obtain time-versus-activity curves for microstructures of interest (that is, those microstructures that govern the organ response to radiation). The integral of each curve

Radionuclide transformations The nuclear or atomic transformations associated with radioactive decay the term 'radionuclide disintegrations' is also used. gives the total number of radionuclide disintegrations for the whole organ and for each subcompartment. With use of these measurements, a series of apportionment factors may be defined for each subcompartment. These preclinically derived apportionment factors may then be used to convert human whole-organ radionuclide disintegrations to the corresponding number of radionuclide disintegrations for each organ subcompartment in humans.

Beyond the aspects highlighted above, there is an extensive literature on RPT dosimetry, including technical aspects ${ }^{53,54,78-86}$, dose-response studies ${ }^{87-91}$ and the rationale for incorporating dosimetry in $\mathrm{RPT}^{92}$.

\section{Cancers targeted by RPT}

In principle, RPT may be applied to any cancer that satisfies the targeting criteria needed for delivery of radionuclides. However, RPT has been investigated for only selected cancers (FIG. 2c). The type of cancer investigated reflects developments related to the available targets, the availability of RPT agents against the targets, and the expertise and clinical investigators at academic institutions.

RPT has had the greatest historical impact for thyroid malignancies and this persists to the present day. Haematological malignancies were investigated starting in the early 1990s and continue to be a subject of interest. RPT for hepatic malignancies and prostate cancer has seen the greatest increase since the 1980s. This increase is consistent with the development of new RPT agents, ${ }^{90}$ Y-loaded microspheres and $\beta$-emitter-labelled and $\alpha$-emitter-labelled small-molecule prostate-specific membrane antigen (PSMA)-targeting constructs, respectively (see later). The FDA-approved $\alpha$-emitter ${ }^{223} \mathrm{Ra}$ has also driven the substantial increase in interest in RPT for prostate cancer. Other solid cancers such as colorectal and breast cancer continue to be of interest but have not had the breakthrough construct development that has driven interest in RPT in hepatic and prostate cancer. Neuroendocrine and somatostatin receptor cancers have been an ongoing subject of investigation, and the RPT agents targeting these cancers have probably reached maturity with the FDA approval of ${ }^{177} \mathrm{Lu}$-labelled DOTATATE.

\section{RPT agents in use and in clinical development}

A number of RPT agents are currently on the market, with many more in development (TABLE 2). These include four $\beta$-particle and five $\alpha$-particle emitters. Lead-212 decays to bismuth-212 and is used as a means to deliver ${ }^{212} \mathrm{Bi}$, an $\alpha$-emitter, without being constrained by its 1-hour half-life. Of the 30 RPT agents listed in TABLE 2, 13 deliver radionuclides that decay by a-particle emission. The interest in $a$-emitters reflects a potential growth area in RPT. Other RPT agents in addition to those discussed below are in preclinical development, but their discussion is beyond the scope of this Review.

RPT can involve the direct delivery of the radioactive element itself ${ }^{93-96}$. A wide variety of 'delivery vehicles' have also been used for RPT (FIG. 3), including small molecules that incorporate the radionuclide ${ }^{19,97,98}$. Radiolabelled peptides and antibodies make up the majority of RPT agents investigated clinically ${ }^{99-106}$. Liposomal or nanoconstruct delivery approaches are being investigated preclinically ${ }^{107,108}$, but these have not yet been tested in human trials. Glass and resin microspheres are relatively well established; these are used in 


\section{Radiohalogen}

Radioactive element in group

17 of the periodic table.

Radiosynovectomy

A treatment for arthritis or

more generally, inflammation that involves injection of

radioactivity, typically as colloidal particles into the synovial cavity. the treatment of hepatocellular carcinoma or hepatic metastases of colorectal cancer ${ }^{109}$ and are administered via the hepatic artery.

The differential retention of different RPT constructs in the tumour is important but difficult to generalize. Antibody-mediated delivery is bivalent and generally leads to long retention, but the long circulating half-life of antibodies leads to greater normal organ, particularly haematological, toxicity. By contrast, small molecules and peptides have the advantage of rapid targeting and clearance, but exhibit typically shorter tumour retention. In all cases, if the agent is internalized and the

\section{Box 2 | Dosimetry scheme for radiopharmaceutical therapy}

The dosimetry formalism presented in BOX 1 entails a number of implicit assumptions that do not apply for dosimetry calculations intended to assess potential toxicity or therapeutic efficacy. In particular, the dosimetry scheme for risk evaluation does not incorporate tumour dosimetry because it relies on reference geometries. The more direct approach of using the measured patient activity distribution from positron emission tomography/computed tomography (CT) or single-photon emission CT/CT images, superimposed over the anatomy as obtained by the CT portion of the imaging scan, has been established. Such voxelized dosimetry approaches use Monte Carlo or point-kernel methods to calculate maps of the spatial distribution of absorbed dose $^{308-310}$. These techniques make it possible to calculate the absorbed dose with regard to actual patient anatomy, including tumours, rather than with regard to a reference, population-averaged, geometry. The generic method is illustrated below. The figure depicts integration over imaging-derived activity values and the use of a point kernel to obtain a map of absorbed doses.

Part a of the figure shows a set of $3 \mathrm{D}$ matrices representing the radioactivity distribution at multiple times $(A(x, y, z, t))$. Integration by volume elements (that is, voxel or a larger discrete structure) is performed over time. This yields a 3D set of images representing the time-integrated activity $(\widetilde{A}(x, y, z))$ in each volume element (represented by $\mathrm{TIA}_{i}$ in part $\mathbf{b}$ of the figure, where TIA is the time-integrated activity). The absorbed dose for a particular volume element, in this example in the kidney, is obtained as the sum of the $\mathrm{TIA}_{i}$ multiplied by a source-to-target distance-dependent absorbed dose per unit TIA (also referred to as a dose point-kernel). The sum over all source volume elements gives the total dose to the target element.

Alternatively, the order could be reversed, with the dose calculation performed on the series of activity images and the integration performed on dose rate rather than activity images. The dose calculation, itself, could be performed directly by Monte Carlo techniques. The latter has the benefit of easily accommodating differences in tissue density and composition. This is particularly important for dose estimates in the vicinity of air or bone tissue interfaces (for example, lung or bone marrow dose calculations).
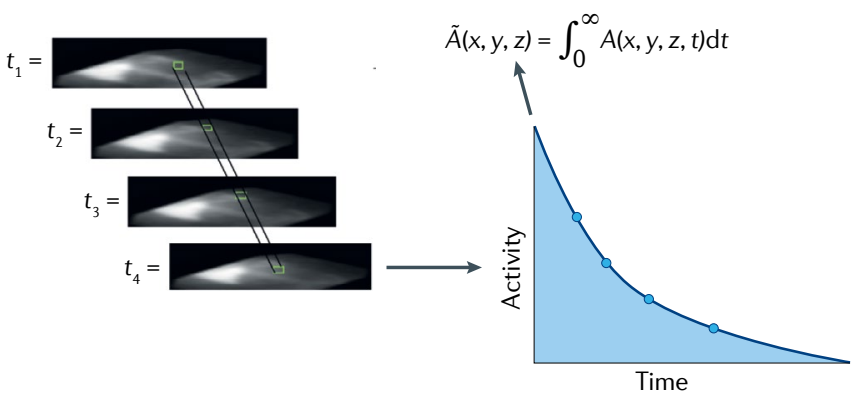

b

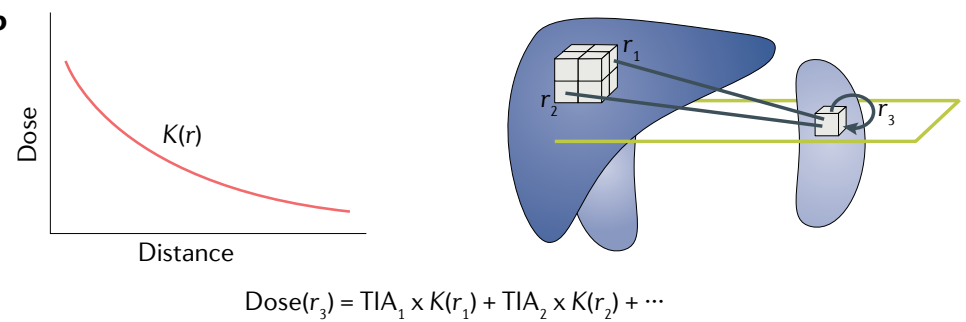

radionuclide retained intracellularly, the target retention time will be very long compared with the clearance kinetics of the agent. Furthermore, in all cases, engineered agents can be designed that optimize tumour retention while increasing clearance kinetics ${ }^{110-116}$.

\section{Unconjugated or chelated radionuclide RPT}

${ }^{[131}$ I]NaI (radioiodine). The prototypical example of RPT is radioiodine treatment of thyroid diseases ${ }^{93,117-120}$. Iodide-131, a $\beta$-particle-emitting radiohalogen with a half-life of 8.02 days, was discovered by John Livingood and Glenn Seaborg following cyclotron bombardment of tellurium ${ }^{121}$. Currently, iodine- 131 is produced for commercial use using a nuclear reactor by irradiating either tellurium-130 $\left({ }^{130} \mathrm{Te}(\mathrm{n}, \gamma)^{131} \mathrm{Te} \rightarrow{ }^{131} \mathrm{I}\right)$ or uranium-235 $\left({ }^{235} \mathrm{U}(\mathrm{n}, \mathrm{f})^{131} \mathrm{I}\right)^{122}$. Radioiodine was initially used to treat hyperthyroidism and thyroid carcinomas ${ }^{123}$. Thyroid follicular cells and differentiated follicular thyroid cancer cells concentrate iodine via a sodium-iodide symporter. The iodide is concentrated in iodine-rich thyroglobulin molecules that are cleaved to produce thyroid hormones. These early discoveries led to the widespread and early use of radioiodine for the treatment of patients with thyroid malignancies. Radioiodine therapy remains the recommended treatment for patients with metastatic differentiated thyroid cancer ${ }^{124}$. Patients with thyroid cancers that originate from cells not involved in concentrating iodine or that are undifferentiated and have lost the molecular machinery to concentrate iodine are not responsive to radioiodine therapy. Patients in the latter category, however, can be successfully treated with radioiodine after differentiation therapy ${ }^{125}$.

${ }^{32} \mathrm{P},{ }^{90} \mathrm{Y}$. Many of unconjugated RPT agents were first developed for radiosynovectomy (note that none of the radiopharmaceuticals is explicitly approved for this scope, but it is an accepted ('established') practice). In Europe these agents continue to be used to treat patients with rheumatoid arthritis and polyarthritis owing to their advantageous faster therapeutic effect and reduced side effects and costs compared with surgical synovectomy ${ }^{5,6}$. Beyond radiosynovectomy, colloidal chromic phosphate (and glass microspheres) containing phosphorus-32 have also been used to treat solid refractory cancers. These radiopharmaceuticals are used to irradiate and kill tumour cells and thus inhibit the growth of malignant disease. The major limitation in these applications is the reliance on radiological guidance for particle deposition (for example, CT, X-ray, ultrasonogram or direct inspection of the surgical field $)^{126}$.

${ }^{223} \mathrm{Ra}$. Radium-223 was the first $\alpha$-emitter approved by the FDA and paved the way to the current development of other $\alpha$-emitting radiopharmaceuticals. Once absorbed into the newly formed bone matrix of osteoblastic metastases, the energetic a-particles emitted by radium-223 can generate irreparable DNA doublestrand breaks in the adjacent osteoblasts and osteoclasts, which can lead to their death. This results in detrimental effects on the neighbouring cancer cells, which then lack their effector of abnormal bone formation, both at a cellular level and at a signalling level (growth factors and 


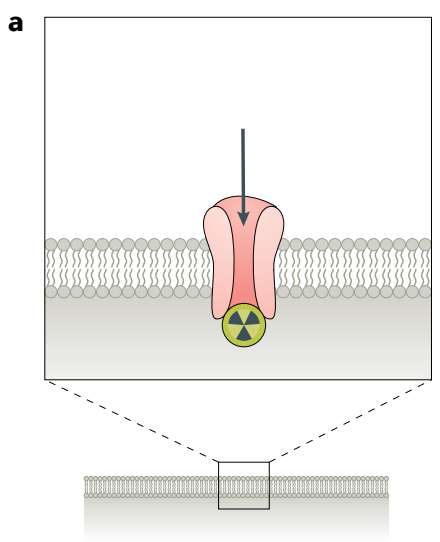

b

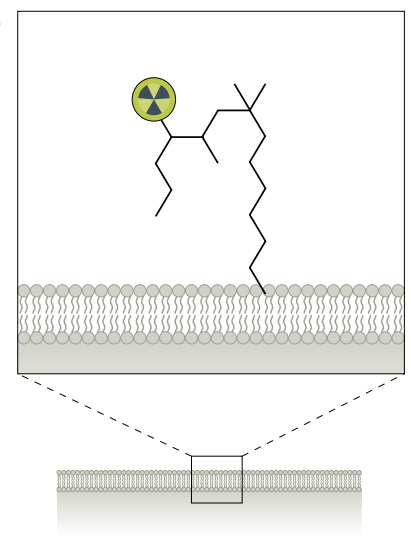

$\mathbf{e}$

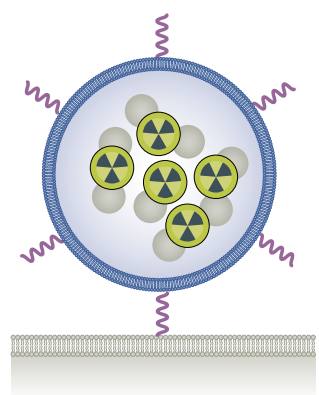

c

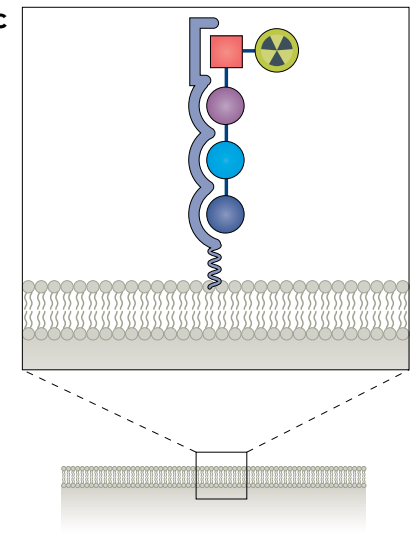

f

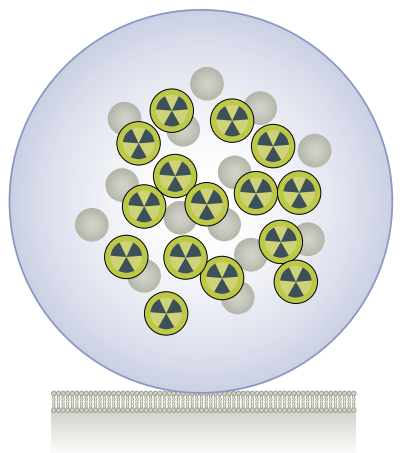

Fig. 3 | Basic RPT constructs used for radiation delivery. The various radiopharmaceutical therapy (RPT) constructs that have been used to deliver radiation are illustrated: radioactive element (part a); small molecule (part b); peptide (part c); antibody (part d); nanoconstruct (part e); microsphere (part f).

immunomodulators), and ultimately in a negative effect on tumour growth. Mechanistic details of this complex interaction are still being evaluated ${ }^{127,128}$.

The numerous clinical applications of radium-223 dichloride in men with metastatic castration-resistant prostate cancer culminated in the phase III placebocontrolled registration ALSYMCA trial, in which radium-223 treatment prolonged survival (14 months versus 11.2 months) when added to best standard of care in men with symptomatic bone metastases in the absence of visceral metastases ${ }^{56}$. To increase the significant but narrow margin of increased overall survival over standard of care, radium-223 is currently being explored in combination with other cytotoxic agents, such as docetaxel (DORA trial, NCT03574571), poly(ADP-ribose) polymerase inhibitors such as olaparib (NCT03317392) and new androgen axis inhibitors such as enzalutamide and abiraterone citrate (PEACE III trial, NCT02194842, and ERA223 trial, NCT02043678, respectively). The first of these trials was recently modified to include bone-protective agents (bisphosphonate) in the treatment regimen ${ }^{129}$, which was found to reduce the incidence of bone fractures. The recommendation was made owing to the observation of an unexpectedly high rate of bone fractures observed in the ERA 223 trial (radium-223 plus abiraterone) ${ }^{130}$, which may have been attributable to the inclusion of prednisone needed to ameliorate the glucocorticoid suppressive effects of abiraterone citrate ${ }^{131}$. Radium-223 is also being explored in combination with immuno-oncology agents such as pembrolizumab (NCT03093428) and in combination with external-beam radiotherapy (RAVENS trial, NCT04037358).

${ }^{153} \mathrm{Sm}$. Samarium-153 ( $t_{1 / 2}=46.3$ hours $)$ is a $\beta$-emitting radionuclide used for palliative treatment in patients with osteoblastic and mixed bone metastases in cancers, such as prostate, breast and other primary cancers ${ }^{132,133}$. ${ }^{153} \mathrm{SmCl}_{3}$ is produced by neutron bombardment of enriched ${ }^{152} \mathrm{Sm}_{2} \mathrm{O}_{3}$, resulting in ${ }^{153} \mathrm{Sm}_{2} \mathrm{O}_{3}$, which is dissolved in hydrochloric acid to yield ${ }^{153} \mathrm{SmCl}_{3}$. Alone, ${ }^{153} \mathrm{SmCl}_{3}$ has poor uptake in bone; however, when chelated with multiple phosphate ligands, the complex accumulates in hydroxyapatite ${ }^{134}$. The affinity of phosphonate for calcium, which is found in rapidly growing bone, is attributed to the accumulation and adsorption of ${ }^{153} \mathrm{Sm}$ in metastatic lesions over normal bone ${ }^{135-137}$. Quadramet is an FDA-approved RPT agent that uses the ethylenediaminetetramethylenephosphonic acid (EDTMP) chelator, binding samarium-153 through six ligands (four phosphate groups and two amines) and forming a six-coordinate complex. An alternative formulation of ${ }^{153} \mathrm{Sm}$ has also been described, ${ }^{153} \mathrm{Sm}$-DOTMP $(1,4,7$, 10-tetraazacyclododecanetetramethylenephosphonic acid), which is thought to have a more favourable chelant-to-metal ratio (1.5:1 versus $273: 1)^{138,139}$. A phase I 
trial of this agent against osteosarcoma has been listed (NCT03612466) but is not yet recruiting.

\section{Small-molecule RPT}

$\left[{ }^{131} I\right] m I B G$. The success of iodide-131 in targeting and treating thyroid disorders and carcinomas encouraged the expansion of its use in a variety of cancers through its incorporation into targeting vectors. For example, iobenguane I-131 is the radioiodinated small-molecule meta-iodobenzylguanidine ([$\left.\left.{ }^{131} \mathrm{I}\right] \mathrm{mIBG}\right)$, an analogue of the adrenergic neutrotransmitter noradrenaline that is used to treat patients with neuroblastomas ${ }^{140-142}$. Iodide-131 can be introduced to targeting vectors as a highly reactive electrophilic iodine compound, allowing rapid iodination of molecules containing activated aromatic groups, or through displacement by nucleophilic attack of the radioiodide ${ }^{143}$. mIBG radiolabelled with high-specific-activity iodine-131 was recently approved by the FDA for the treatment of adult and paediatric patients aged 12 years or older with unresectable metastatic phaeochromocytoma or paraganglioma. No FDAapproved therapy was available for these conditions before approval of this agent. Use of this agent requires a positive $\mathrm{mIBG}$ imaging scan, standard, weight-based therapeutic dosing and the application of a process for individualized dosimetry using a pretreatment tracer study to calculate absorbed doses for normal organs. Normal organ dosimetry is used to adjust the activity administered so that the organ doses are below specified threshold levels. FDA approval of this agent was based on the substantial pre-existing experience with $\left[{ }^{131} \mathrm{I}\right] \mathrm{mIBG}^{144-151}$ and on a recent phase I study which yielded 1-year and 2-year overall survival of $85.7 \%$ and $61.9 \%$, respectively, in 21 patients treated with the maximum tolerated dose $\mathrm{e}^{152}$. Clinical trials using this agent are ongoing (NCT03561259 and NCT02378428).

PSMA and folate receptor ligands. The past decade has seen increasing use of small-molecule radiotherapeutics targeting receptors such as PSMA receptor and folate receptor (FR). Small-molecule PSMA inhibitors have been modified to deliver radiotherapeutic nuclides in prostate cancer as well as other cancers owing to the expression of PSMA on the neovasculature of tumours. PSMA inhibitors are designed to mimic its substrates, $\gamma$-glutamyl folic acid derivatives and the neuropeptide $\mathrm{N}$-acetylaspartylglutamate ${ }^{153,154}$. These small-molecule RPT agents are either urea-based inhibitors (for example, ${ }^{177} \mathrm{Lu}$-labelled PSMA-R2 and ${ }^{177} \mathrm{Lu}$-labelled PSMA-617) ${ }^{155-162}$ or phosphoramidate-based inhibitors (for example, ${ }^{177} \mathrm{Lu}$-labelled CTT-1403) modified to deliver therapeutic radionuclides ${ }^{163-165}$. The urea and phosphoramidate functionalities interact with zinc(II) located in the PSMA active site; in addition, these small molecules are designed to interact with the $S 1$ glutamate pocket as well as the entrance funnel of PSMA (FIG.4a,b). The entrance funnel allows a wide variation of modifications to present a pendant group or a chelator for radiolabelling with therapeutic and imaging radionuclides. All three agents are currently in clinical trials: NCT03490838, NCT03511664 and NCT03822871, respectively (TABLE 2).
${ }^{177} \mathrm{Lu}$-labelled PSMA-617 is currently in a multicentre (84 sites), phase III randomized trial (VISION). In a previous prospective single-centre phase II trial in men with metastatic castration-resistant prostate cancer in whom standard therapies had failed, patients who showed high expression of the PSMA target by $\left({ }^{68} \mathrm{Ga}\right.$-labelled PSMA-11) PET yielded favourable responses; patients with high $\left[{ }^{18} \mathrm{~F}\right]$ fluorodeoxyglucose (FDG) uptake in low-PSMA-uptake lesions were excluded. Of 43 enrolled patients, 30 met the PSMA and FDG imaging criteria to advance to therapy. At 3 months after therapy, reductions in tumour dimensions (by CT) and PSMA uptake and metabolic activity (by PET) were observed in approximately $40 \%$ of treated patients ${ }^{166}$. The imaging-based screening criteria used in this study highlighted the ability to image the distribution of the therapeutic target - a feature that is integral to RPT. Although the theranostic (that is, imaging-based patient selection) used in this trial has been discussed as providing a highly favourable patient population and potentially excluding patients who may have benefited from the treatment ${ }^{167,168}$, the percentage of screened patients who were treated was higher than for most genomics-based screening criteria that are typically used in trials of biologic agents ${ }^{169}$.

The ${ }^{177} \mathrm{Lu}$-labelled PSMA-R2 ligand is in a phase I/II multicentre dose-escalation study. The eligibility criteria for this study include a positive ${ }^{68} \mathrm{Ga}$-labelled PSMA-R2 PET scan; in contrast to PSMA-617, lesion metabolism by FDG PET is not a screening criterion. Preclinical comparison of these two agents suggests similar efficacy ${ }^{170}$.

The ${ }^{177} \mathrm{Lu}$-labelled CTT-1403 anti-PSMA ligand is an irreversible phosphoramidate-based PSMA inhibitor that includes an albumin-binding motif ${ }^{171,172}$. This agent is in a first-in-human phase I dose-escalation trial. As with the two urea-based agents described earlier, CTT1403 also incorporates a theranostic paradigm using a companion diagnostic, CTT-1057, which is a ${ }^{18} \mathrm{~F}$-labelled PET agent.

Recent reviews provide more background regarding PSMA-targeting ligands ${ }^{173,174}$.

FR is overexpressed in a variety of cancers with limited expression in normal tissue; expression on the luminal surface of the kidneys is the greatest concern for FR-targeted radiotherapeutics ${ }^{175,176}$. Similarly to PSMA-targeted radiotherapy, FR-targeted radiotherapeutics have been developed based on the substrates of FR - specifically folate. Derivatives of folate have been developed for delivery of radionuclides for imaging and therapy (FIG. 4C); however, currently the FR-targeted radiotherapeutics have not been translated to clinical studies $^{177-181}$.

Phospholipid ether analogues. Early observations of a difference in the membrane composition of neoplastic cells compared with normal cells ${ }^{182,183}$ led to the development of radioiodinated phospholipid ether constructs that showed rapid and persistent tumour accumulation $^{184,185}$. The localization and clearance properties of these low-molecular-weight agents depend on the length of the alkyl chain and they localize to tumours not by a specific receptor but by an overabundance of 


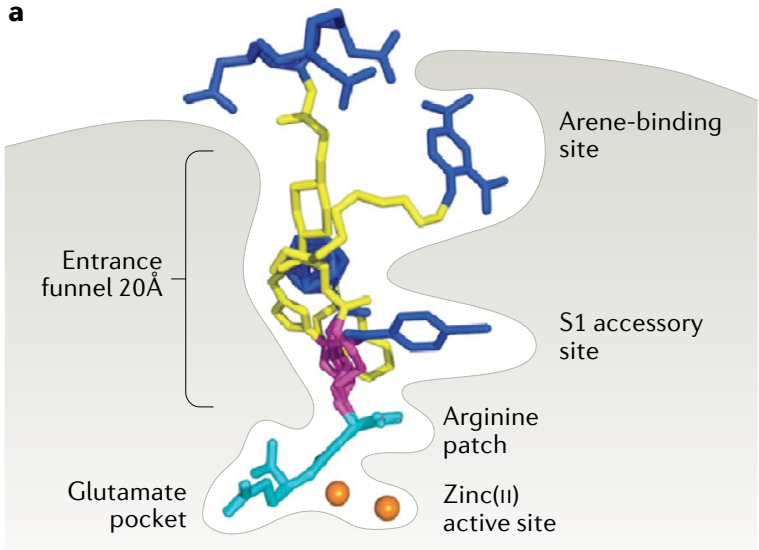

b

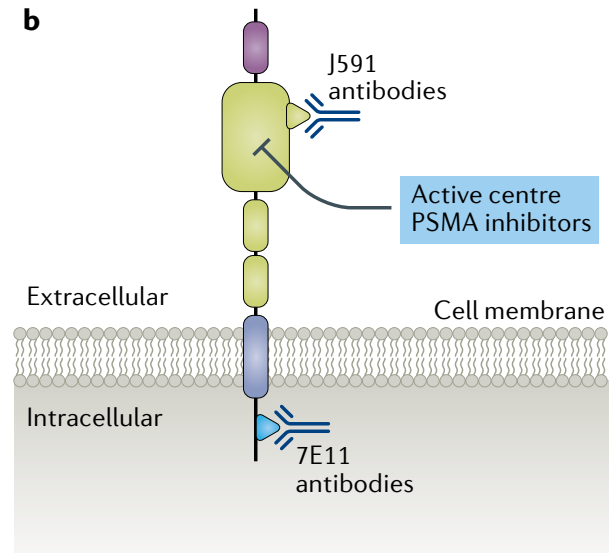

c

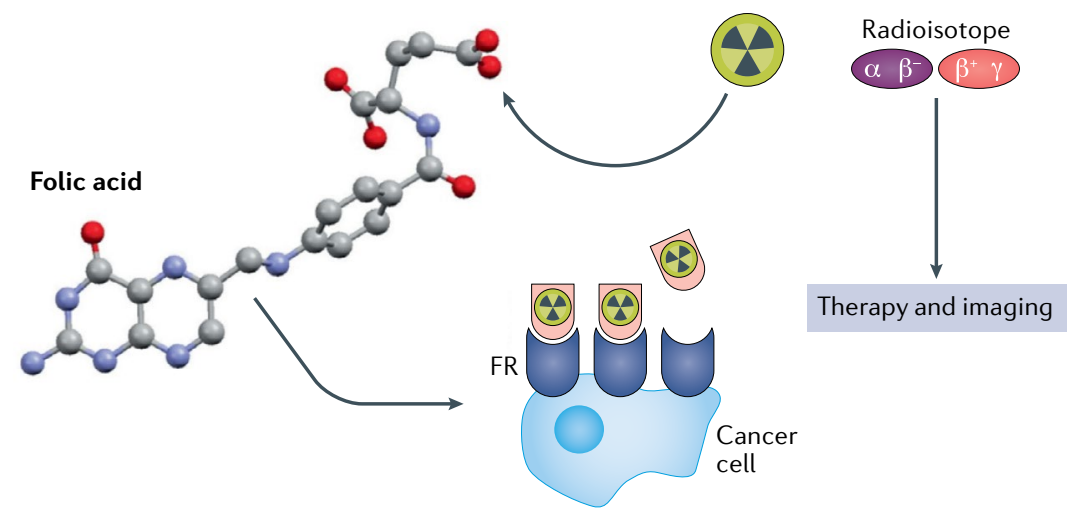

Fig. 4 | PSMA and folate receptor RPT. a | Prostate-specific membrane antigen (PSMA) inhibitor binding pocket. b | PSMA receptor showing sites of small-molecule and anti-PSMA interactions $\mathbf{c}$ Folate receptor (FR) radiopharmaceutical therapy (RPT). Conjugation of an $\alpha$-emitter or a $\beta^{-}$-emitter for therapy or a positron $\left(\beta^{+}\right)$-emitter or a $\gamma^{-e m i t t e r ~ f o r ~ p o s i t r o n ~ e m i s s i o n ~}$ tomography or single-photon emission computed tomography, respectively. This is followed by FR targeting. Part a: this research was originally published in JNM. Kopka, K. et al. Glu-ureido-based inhibitors of prostate-specific membrane antigen: lessons learned during the development of a novel class of low-molecular-weight theranostic radiotracers. J. Nucl. Med. 58, 17S-26S (2017), CSNMMI (REF. ${ }^{302}$ ). Part b adapted from REF. ${ }^{303}$, Springer Nature Limited. Part c, this research was originally published in JNM. Müller, C. et al. Folic acid conjugates for nuclear imaging of folate receptor-positive cancer. J. Nucl. Med. 52, 1-4 (2011), (SNMMI (REF. ${ }^{304}$ ).

lipid rafts present on cancer cell membranes ${ }^{186,187}$. These agents have been used for both cancer imaging and cancer therapy ${ }^{65,188-191}$. As pan-cancer RPT agents they are under clinical trial investigation for a wide spectrum of haematological malignancies and solid tumours (NCT03478462, NCT04105543, NCT02952508, NCT02952508 and NCT01495663).

\section{Peptide and antibody RPT}

Peptide receptor radionuclide therapy (PRRT) with radiolabelled somatostatin analogue peptides (FIG. 5) was developed in the early 1990s as the next logical step of the somatostatin receptor-mediated radionuclide localization of neuroendocrine tumours (NETs).

After initial experiences with the diagnostic radionuclide indium-111 (( ${ }^{111} \mathrm{In}-\mathrm{DTPA}^{0}$-D-Phe $\left.{ }^{1}\right)$-octreotide or ${ }^{111}$ In-pentetreotide), in an attempt to exploit the Auger and conversion electron emissions of indium-111, the past two decades have seen the use of the more suitable $\beta$-emitters, yttrium-90, and, later, lutetium-177, with their longer-range emissions (see below). This therapeutic strategy has been accepted as an effective therapeutic modality in the treatment of inoperable or metastatic gastroenteropancreatic and bronchopulmonary NETs, as well as phaeochromocytomas and paragangliomas. The two most commonly used radiopeptides for PRRT, ${ }^{90} \mathrm{Y}$-octreotide $\left({ }^{90} \mathrm{Y}\right.$-DOTA-Tyr ${ }^{3}$-octreotide) and ${ }^{177} \mathrm{Lu}$-octreotate $\left({ }^{177} \mathrm{Lu}\right.$-DOTA-Tyr ${ }^{3} \mathrm{Thr}^{8}$-octreotide), produce disease-control rates of $68-94 \%\left(\right.$ REFS $\left.^{192,193}\right)$. These studies culminated in the phase III registration trial NETTER-1, which demonstrated significantly longer progression-free survival in patients with small-bowel NETs treated with ${ }^{177} \mathrm{Lu}$-octreotate plus $30 \mathrm{mg}$ long-acting-release octreotide than in those treated with $60 \mathrm{mg}$ long-acting-release octreotide ${ }^{194}$. In addition to tumour responses, symptomatic and quality of life improvement is commonly observed and was confirmed in the NETTER-1 trial post-hoc analyses $^{192,195}$. These results compare favourably with the efficacy of 'cold' somatostatin analogues, chemotherapy and 'targeted' therapies such as everolimus and sunitinib therapies ${ }^{196}$. A major advantage of PRRT over conventional treatments is the possibility to identify and quantify the target, the somatostatin receptor, before 


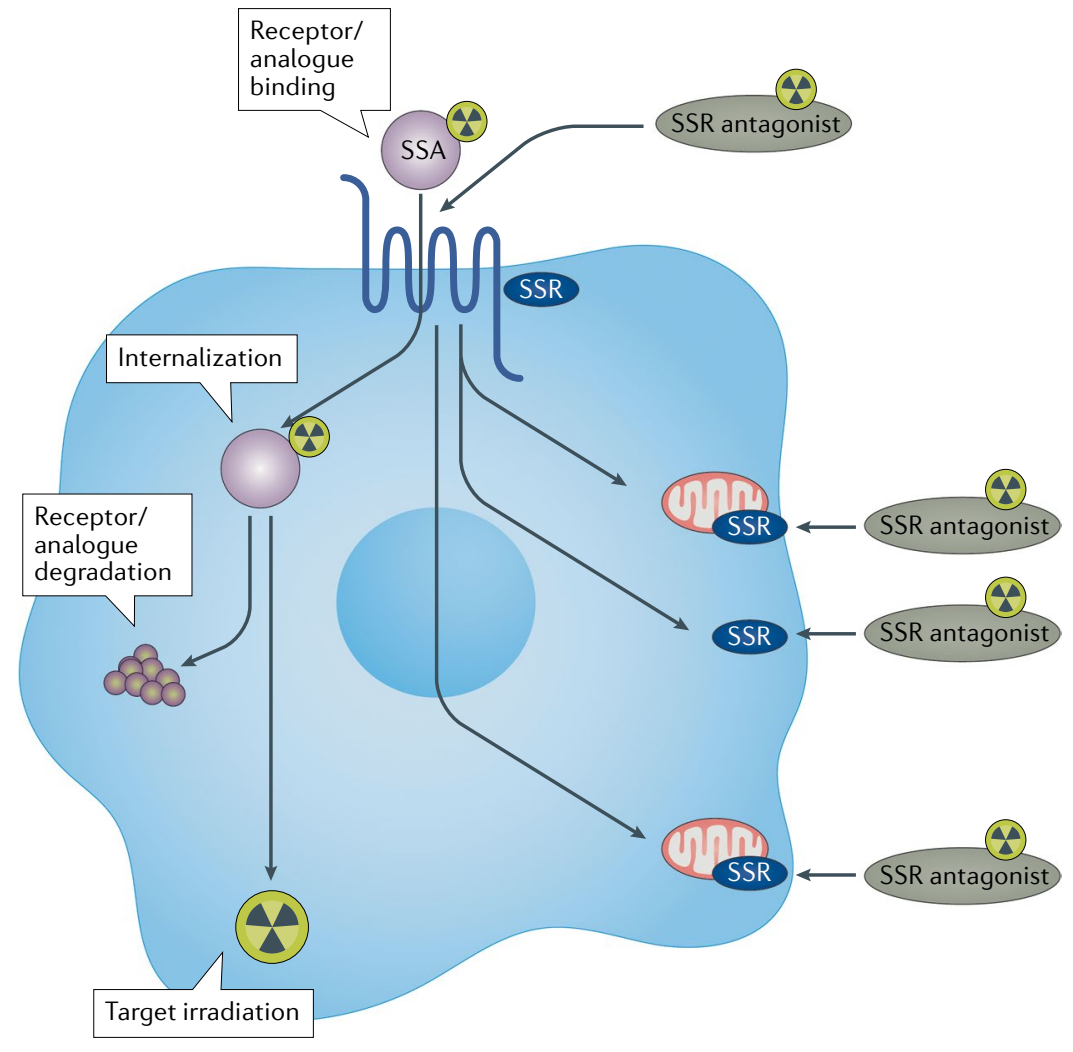

Fig. 5 | Mechanism of action of peptide receptor radionuclide therapy. The somatostatin analogue (SSA), generally an octreotide derivative, an agonist, is linked to a DOTA chelator, which contains the radionuclide. After binding to the membrane somatostatin receptor (SSR), the radiopeptide is internalized and is transported into the intracellular receptor-recycling compartment. Recently introduced SSR antagonists have overturned this principle, having proven to have significantly lower internalization but greater binding, owing to recruitment of inactive SSRs on the surface of the neuroendocrine tumour cell. Adapted with permission from REF. ${ }^{192}$, Elsevier.

initiation of therapy, in the most classic of the theranostic approaches. Recently the PRRT strategy against somatostatin receptor-positive cancer was extended to the delivery of the $\alpha$-emitter lead-212 (NCT03466216) ${ }^{197-199}$.

In general, PRRT is well tolerated with only low to moderate toxic effects on the bone marrow and kidneys in most patients. There is a mild loss of renal function, and subacute and transient haematological toxicity is mild in approximately $90 \%$ of cases. Myelodysplastic syndrome and sporadic instances of leukaemias have been reported in approximately $2 \%$ of cases ${ }^{192,200}$.

PRRT with radiolabelled octreotides constitutes a paradigm that in its 25 years of use has still not been outperformed by the introduction of other agents. However, different strategies aimed at improving the outcomes have been proposed. The recently introduced somatostatin receptor antagonists, which are able to recruit also inactive membrane somatostatin receptors, result in a higher irradiation of the NET cell, despite the lower internalization. Preliminary clinical studies with ${ }^{177} \mathrm{Lu}$-DOTA-JR11 demonstrated an overall response rate of $45 \%$ with a disease-control rate of $85 \%$ with only two therapeutic cycles, as opposed to the standard four cycles of the 'conventional' PRRT ${ }^{201}$. An ongoing phase I/II multicentre study (NCT02592707) will define the optimal therapeutic dose/schedule to be adopted in an upcoming phase III trial. Lastly, octreotide derivatives labelled with $\alpha$-emitters, such as lead-212 (NCT03466216) or actinium-225 are being explored to increase therapeutic efficacy, especially in patients who are refractory to conventional PRRT.

Bombesin analogue peptides. Following the success of the somatostatin receptor theranostics, other G proteincoupled receptors with similar binding properties have been explored, including the mammalian gastrinreleasing peptide (or amphibian bombesin) receptors, BB1 (NMBR), BB2 (GRPR) and BB3 (BRS3) 202 . These receptors are overexpressed in several cancers, mainly in prostate and breast cancers ${ }^{203-208}$.

After initial experiences with agonists, such as the ${ }^{68} \mathrm{Ga} /{ }^{177} \mathrm{Lu}$-labelled AMBA theranostic pair, burdened by a multitude of symptoms related to receptor activation and by poor plasma stability ${ }^{209}$, clinical trials have proceeded with antagonists, owing to their superior targeting properties and their neutral pharmacologic action and resistance to peripheral peptidase degradation ${ }^{210}$. Multiple antagonists have been developed for imaging, such as ${ }^{68} \mathrm{Ga}$-labelled RM2 (formerly BAY 867548), ${ }^{68} \mathrm{Ga}$-labelled RM26 and ${ }^{68} \mathrm{Ga}$-labelled NeoBOMB1 $\left(\mathrm{REF}^{203}\right)$. Therapeutic experience is, however, limited to ${ }^{177} \mathrm{Lu}$-labelled NeoBOMB1 and ${ }^{177} \mathrm{Lu}$-labelled RM2. The former has been studied in PC-3 tumour-xenografted nude mice, demonstrating high tumour uptake and favourable pharmacokinetics, including limited pancreatic uptake, which makes it a suitable candidate for clinical applications ${ }^{211}$.

The dosimetry of the GRPR antagonist ${ }^{177} \mathrm{Lu}$-labelled RM2 was recently studied in individuals with metastatic castration-resistant prostate cancer. The analysis showed high tumour doses $(6.20 \pm 3.00 \mathrm{~Gy} / \mathrm{GBq})$, with rapid clearance from normal organs, including the pancreas, which are promising attributes for therapeutic applications $^{212}$.

Antibody-based RPT. The IgG antibody class has been used almost exclusively for RPT. IgG antibodies are proteins with a molecular weight of 150,000 and which have a long biological half-life in the circulation (2-5 days, depending on their isotype and structure) and are eventually catabolized by the liver and elements of the reticuloendothelial system ${ }^{213,214}$. The ability to generate antibodies possessing well-defined reactivity characteristics against selected antigens (that is, monoclonal antibodies ${ }^{215}$ ) was a precondition for the use of antibodies as viable radionuclide delivery vehicles. Haematological and lymphoid malignancies have dominated RPT investigations. Antibodies to cell-surface differentiation antigens (for example, CD33, CD20, CD45 and CD37) were available for radiolabelling and clinical investigations (FIG. 6). These early studies used the readily available radionuclide ${ }^{131} \mathrm{I}$, which permitted (1) biodistribution of the radioiodinated antibodies to be imaged using SPECT ${ }^{216,217}$ and (2) therapy ${ }^{218-220}$. Radiolabelled antibodies in this class of targets were used either as molecularly targeted replacements for total-body irradiation in preparation for bone marrow transplantation 
(NCT02665065) ${ }^{221-228}$ or for direct targeting of lineages that exhibit uncontrolled proliferation ${ }^{229-231}$. More recently, the $\alpha$-emitter astatine- 211 is being investigated with anti-CD45 as a lower-toxicity alternative to iodine-131 or radiotherapy for bone marrow ablation in preparation for transplantation ${ }^{232,233}$.

Escalating doses of the iodinated antibody M195 (anti-CD33) were used to treat ten patients with acute myelogenous leukaemia in a phase I clinical trial ${ }^{234}$. The appending of iodine-131 to the M195 antibody allowed detailed pharmacokinetic and dosimetric studies to be performed as well as serial blood and bone marrow sampling and whole-body $\gamma$-camera imaging. Disease in the bone marrow was specifically and clearly imaged starting within hours after injection. The radioiodinated M195 was rapidly modulated and internalized into target cells in vivo. Importantly, a rapid, specific and quantitative delivery to the bone marrow and the efficient internalization of M195 into target cells in vivo suggested that the delivery of $\beta$-emitting and $\alpha$-emitting radionuclides was feasible. Humanization of M195 (HuM195, lintuzumab) and access to a-particle-emitting bismuth-213 and actinium-225 and $\beta$-particle-emitting yttrium-90 radionuclides expanded the warheads available to the anti-CD33 armament $^{235-240}$. The pioneering studies using iodine-131 advanced the utility of the humanized anti-CD33 with more potent and shorter-ranged energy emissions because the pharmacokinetics and biology of the system were well understood. Potent antileukaemic responses have been observed with both of the a-particleemitting lintuzumab drugs with minimal off-target toxic effects ${ }^{239,241-243}$. Actinium-225-labelled anti-CD38 targeting multiple myeloma has also been studied ${ }^{244,245}$.

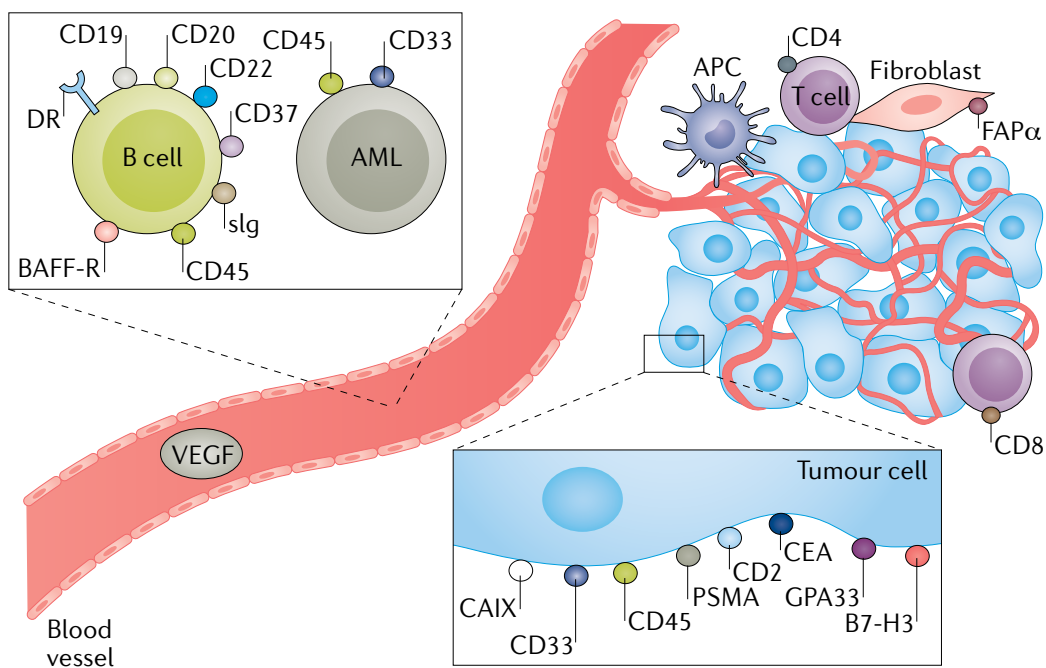

Fig. 6 | Target antigens that have been used in antibody-based radiopharmaceutical therapy. Antibodies to a variety of tumour-associated targets may be raised, including leukaemia-associated and lymphoma-associated targets (for example, CD20, CD45 and CD33), targets expressed on solid-tumour cancer cells (for example, carcinoembryonic antigen (CEA), prostate-specific membrane antigen (PSMA) and GD2) and targets expressed on their supporting microenvironment (for example, fibroblast activation protein- $\alpha(\mathrm{FAP} \alpha))$. AML, acute myelogenous leukaemia; APC, antigen-presenting cell; BAFF-R, B cell-activating factor receptor; CAIX, carbonic anhydrase 9; DR, death receptor; slg, secretory immunoglobulins. Adapted from REF. ${ }^{305}$, Springer Nature Limited.
This $\alpha$-emitter is also being investigated against solid tumours that express insulin-like growth factor receptor type 1 (NCT03746431) ${ }^{246}$. Several antibodies for delivery of a low-dose-rate $\alpha$-particle emitter, thorium-227, are being investigated ${ }^{247}$. Thorium-227-conjugated antibodies targeting mesothelin ${ }^{248,249}$, PSMA ${ }^{250,251}$, and CD22 (REF. ${ }^{252}$ ) are currently in phase I clinical trials (NCT03507452, NCT03724747 and NCT02581878, respectively). An anti-HER2/neu conjugate is under preclinical investigation. This conjugate has demonstrated substantially greater antitumour efficacy compared with a toxin (T-DM1) anti-HER2/neu conjugate $\mathrm{e}^{253-255}$. It has also demonstrated synergy in combination with olaparib in a BRCA2-deficient xenograft model ${ }^{256}$

Lymphoma radionuclide therapy followed a similar trajectory to that of acute myelogenous leukaemia described earlier. Rituximab (anti-CD20) was developed to treat relapsed and refractory follicular low-grade non-Hodgkin lymphoma (NHL). When it was used as a single agent, response rates of $50-70 \%$ in follicular lymphoma and $15-50 \%$ in small B cell lymphoma were reported. These indolent lymphomas are characteristically radiosensitive; however, external-beam radiation is limited by toxic effects on normal tissues. Delivery of the radioactive emission source specifically to cancer cells is accomplished via the monoclonal antibody delivery vehicle, providing a potent dose of targeted radiation therapy specifically to tumour cells that express the desired antigen ${ }^{257-260}$.

There are two intact antibodies directed against the CD20 antigen which have been conjugated to radionuclides. Ibritumomab tiuxetan conjugated to yttrium-90 yielded Zevalin (Schering AG, Berlin, Germany) and tositumomab conjugated to iodine-131 yielded Bexxar (Corixa Corp, Seattle, WA, USA). Both ${ }^{90}$ Y-ibritumomab and ${ }^{131} \mathrm{I}$-tositumomab have been compared with their respective unlabelled antibody counterparts in randomized trials.

In a phase III study of patients with relapsed CD20positive NHL, those who received ${ }^{90} \mathrm{Y}$-ibritumomab had a higher overall response rate than those who received the 'cold' unlabelled rituximab (response rate of $80 \%$ compared with $56 \%$, respectively $)^{261}$. Complete response rates of $30 \%$ and $16 \%$, respectively, were reported. Reversible myelosuppression was associated with ${ }^{90} \mathrm{Y}$-ibritumomab therapy. In addition, in a randomized trial designed to treat relapsed low-grade or transformed indolent NHL, patients receiving the 'hot' ${ }^{131}$ I-labelled tositumomab drug exhibited response rates of 55\%, while those receiving 'cold' unlabelled tositumomab exhibited only a $33 \%$ response rate. The complete response rates reported were $17 \%$ and $8 \%$, respectively ${ }^{262}$.

The amount of ${ }^{131}$ I-tositumomab prescribed to patients was determined by assessing the whole-body clearance rate, so that the amount administered was adjusted to deliver the same whole-body absorbed dose in all treated patients ${ }^{263}$, making it the first RPT agent whose package insert specified an absorbed dose-based treatment planning procedure. Such an approach was, in part, necessitated because the radioiodine in iodine-131-labelled antibodies is cleaved 
(due to dehalogenation) from the antibody if the radiolabelled antibody construct is internalized.

As mentioned already, there are a number of antigenic targets for antibody-based RPT for solid tumours (FIG. 6). However, antibody-mediated RPT for solid tumours has not provided the clinical impact of antibody-mediated RPT for haematological and lymphoid malignancies. This may be explained by the substantially greater absorbed dose required to effectively treat solid cancers - 50-80 Gy compared with as low as $3 \mathrm{~Gy}$ for some lymphomas ${ }^{103}$. Radiolabelled antibodies must overcome a number of barriers before they can effectively irradiate solid tumour targets. They must extravasate and diffuse across an interstitial fluid space that is characterized by pressure gradients opposing macromolecular transport ${ }^{264-266}$ and penetrate throughout antigen-positive tumours, wherein a high antigen concentration itself presents a binding-site barrier ${ }^{267-269}$. These barriers, along with the long circulation half-life of antibodies, lead to high bone marrow absorbed doses and insufficient dose delivery to tumours. A number of strategies that retain the exquisite specificity and high binding affinity of antibodies have been examined to overcome these limitations ${ }^{270,271}$. The most widely investigated of these is 'pretargeting', in which delivery of the radionuclide is temporally dissociated from tumour targeting. The original pretargeting paradigm involved administration of a radiolabel-free streptavidin-conjugated antibody. This was followed by administration of lower molecular weight biotin which is radiolabelled ${ }^{272}$. The initial construct localizes to tumours without irradiating the bone marrow. The subsequent radiolabelled biotin has a lower molecular weight and is not susceptible to the pharmacokinetics and barriers of intact radiolabelled antibody delivery. Antibody engineering has eliminated the requirement for bacterial-derived elements (with their attendant immunogenicity), and a large number of novel approaches have been developed that are founded on the basic concept of temporal dissociation ${ }^{273}$.

\section{Nanoconstruct and microsphere RPT}

Yttrium-90 radioembolization is a technique that targets radiolabelled microspheres to liver tumours associated with unresectable hepatocellular carcinomas or metastatic liver tumours from primary colorectal cancer. Liver tumours derive their blood supply from the hepatic artery, whereas the normal liver derives its blood supply from the portal vein, allowing targeted delivery of ${ }^{90}$ Y-loaded microspheres via intra-arterial injection ${ }^{274-276}$. The commercially available ${ }^{90} \mathrm{Y}$-loaded microspheres are either glass based (TheraSphere) or resin based (SIR-Spheres), differing in size, number of microspheres injected and activity per microsphere ${ }^{277,278}$. Both agents were approved by the FDA and are marketed as devices. We include these as RPT agents because they better fit the broad definition of RPT agents provided earlier. The findings of studies comparing ${ }^{90} \mathrm{Y}$-loaded glass-based and resin-based microspheres are conflicting and further investigation is needed. For example, while one study in patients with unresectable hepatocellular carcinomas found a significantly higher overall survival for treatment with ${ }^{90} \mathrm{Y}$-loaded glass microspheres compared with ${ }^{90}$ Y-loaded resin microspheres ${ }^{279}$, another study found a similar outcome in terms of progression-free survival and overall survival between patients treated with ${ }^{90}$ Y-loaded glass-based microspheres and patients treated with ${ }^{90} \mathrm{Y}$-loaded resin-based microspheres ${ }^{278}$. Additional transarterial radiotherapeutics are being explored, including phosphorus-32 glass microspheres

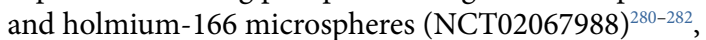
as well as ${ }^{131} \mathrm{I}$-labelled or ${ }^{188} \mathrm{Re}$-labelled iodized oil $^{282-284}$. Initial clinical trials of ${ }^{131}$ I-labelled iodized oil ( ${ }^{131}$ I-labelled Lipiodol) were completed in the late 1980s/ early $1990 \mathrm{~s}^{285-288}$, and clinical investigations of this treatment modality continued until 2013 (NCT00116454, NCT00870558 and NCT00027768). Administration of ${ }^{131}$ I-labelled Lipiodol in the adjuvant setting, after resection or radiofrequency ablation for hepatocellular carcinoma, yielded a 6-month increase in recurrencefree survival and a 24-month increase in median overall survival $^{289}$. In a prospective randomized 43 -patient trial, adjuvant treatment of patients with hepatocellular carcinoma led to a significant increase in overall survival at 3 years of $86.4 \%$ in the treated group versus $46.3 \%$ in the control group ${ }^{290}$. At both the 5-year follow-up and the 10-year follow-up, actuarial overall survival in the treated group was statistically significantly greater than in the control group ( $66.7 \%$ versus $36.4 \%$, respectively, and $52.4 \%$ versus $27.3 \%$, respectively). The difference in overall survival lost statistical significance 8 years after randomization ${ }^{291}$.

\section{Challenges and considerations in RPT}

RPT has proven to be an effective cancer treatment when other standard therapeutic approaches have failed. However, despite more than 40 years of clinical investigation, RPT has not become a part of the cancer treatment armamentarium in the same way as other therapies ${ }^{292}$. 'Targeted' cancer therapies are associated with clinical trial failure rates of $97 \%$ (REF. ${ }^{1}$ ), partly because the agents targeted a pathway that was not involved in promoting the cancer phenotype ${ }^{2}$. By contrast, RPT has been unsuccessful owing to a failure to adopt and rigorously evaluate this treatment modality, which may be explained in part by the multidisciplinary nature of the treatment.

Additional challenges facing the development and application of RPT include public perception and fear of radioactivity as well as the perceived complexity of the treatment. Until very recently, the $>40$ years of experience with these agents was largely ignored or presented as a burdensome multidisciplinary endeavour in the medical literature. A 2007 review of the management of painful bone metastases ${ }^{293}$ highlights this, implying that the efficacy, low toxicity, minimal side effects and non-addictiveness of RPT for bone pain palliation is trumped by the complexity and need for a multidisciplinary implementation. The lack of a medical constituency for RPT suggests the need for a new specialty or subspecialty to provide the multidisciplinary training needed to safely and effectively administer RPT agents to patients and subsequently manage them. Such a specialty or subspecialty would require training in nuclear medicine, radiation oncology and also general oncology. 
As delivery of radiation is involved, the participation of medical physicists familiar with both imaging and radionuclide dosimetry is important.

Radionuclide supply, in particular for a-particleemitting radionuclides (such as actinium-225), is considered a potential obstacle for growth of the field. Technological efforts to address the radionuclide supply are ongoing by both the public sector ${ }^{294,295}$ and the private sector. These developments suggest that supply issues are transient technical issues that will be resolved with greater investment if RPT is adopted as a mainstream cancer therapy.

Assuming that the early dramatic results obtained with $\alpha$-particle RPT continue to be borne out in rigorous clinical trials, the growth of $\alpha$-emitter RPT is likely to accelerate. However, the development of biological resistance to these agents - likely due to outgrowth of cancer cells with low or no target expression - must be considered. Accordingly, combination treatment will be necessary. Identifying the optimal combination cancer therapy is currently largely a trial-and-error effort. By incorporating molecular imaging to assess the impact of other therapeutics on RPT delivery and calculation of the target and normal tissue absorbed doses, the trial-and-error effort needed to optimize combination therapy for RPT agents can be substantially reduced. Furthermore, preclinical models to assess changes in radiosensitivity arising from combination therapy can also be used for optimization. In contrast to biologics or chemotherapeutics, both radiation delivery and the biological response to radiation may be mathematically modelled and used to understand the parameters of a treatment that are most important in influencing efficacy and toxicity ${ }^{296}$. In this sense, the trial-and-error process can be further attenuated by modelling to reduce the 'parameter space' that needs to be clinically investigated. Finally, as a radiation delivery modality, one may envision widespread adoption of treatment planning that combines RPT with external-beam radiotherapy; the former to target disseminated cancer and the latter to target bulky disease that is less effectively treated by RPT. Such a combination strategy would expand patient eligibility for both RPT and external-beam radiotherapy.

\section{Outlook}

The systemic delivery of short-ranged potent radiation is an effective approach to treating patients with cancer, exhibiting a number of advantages over current therapeutic strategies. These advantages include the ability to image and calculate quantities that directly impact efficacy and potential toxicity such as absorbed dose, the ability to deliver radiation that is impervious to almost all conventional resistance mechanisms and the ability to combine RPT with radiotherapy in a rational and absorbed dose-driven manner so as to reduce the level of empiricism in clinical trials. The field of RPT has been active and growing for nearly 40 years, and is attracting a high level of recognition and commercial interest (TABLE 2).

Aside from establishing trained practitioners, the future growth of RPT will be fuelled by continued discovery of more-specific tumour-associated targets, improvements in radiochemistry, increased and low-cost availability of radionuclides (particularly a-particle emitters) and expanded preclinical and clinical investigation of combination therapy with agents and treatment modalities that are complementary to RPT. The ability to image and quantitatively characterize the likely biological outcome of RPT through dosimetry and treatment planning is a major and unique strength of this treatment approach.

The question to be addressed is how does the field strike the right balance between using those features of RPT - imaging, dosimetry and treatment planning that can help guide and optimize patient treatment and that provide an advantage over other cancer therapies versus the more expedient approach of adopting a chemotherapy dosing paradigm? The former is seen as too complicated, while the latter is already in use, is thought to work well enough and has already introduced commercially viable and beneficial agents to patients. The answer lies in early-stage clinical trials that incorporate imaging and dosimetry so that the value of these unique aspects of RPT may be rigorously evaluated and compared with existing therapeutic approaches.

Published online 29 July 2020
1. Wong, C. H., Siah, K. W. \& Lo, A. W. Estimation of clinical trial success rates and related parameters. Biostatistics 20, 273-286 (2018).

2. Lin, A. et al. Off-target toxicity is a common mechanism of action of cancer drugs undergoing clinical trials. Sci. Transl Med. 11, eaaw8412 (2019).

3. Gill, M. R., Falzone, N., Du, Y. \& Vallis, K. A. Targeted radionuclide therapy in combined-modality regimens. Lancet Oncol. 18, e414-e423 (2017).

4. Dolgin, E. Radioactive drugs emerge from the shadows to storm the market. Nat. Biotechnol. 36, 1125-1127 (2018).

5. Knut, L. Radiosynovectomy in the therapeutic management of arthritis. World J. Nucl. Med. 14, 10-15 (2015).

6. Kresnik, E. In: Local Treatment of Inflammatory Join Diseases: Benefits and Risks (eds Kampen, W. U., \& Fischer, M.) 81-93 (Springer International Publishing, 2015).

7. Bentzen, S. M. et al. Quantitative analyses of normal tissue effects in the clinic (QUANTEC): an introduction to the scientific issues. Int. J. Radiat. Oncol. Biol. Phys. 76, S3-S9 (2010).

Summary of radiation dose versus response data from radiotherapy experience.
8. Dale, R. \& Carabe-Fernandez, A. The radiobiology of conventional radiotherapy and its application to radionuclide therapy. Cancer Biother. Radiopharm. 20, 47-51 (2005).

9. Amro, H., Wilderman, S. J., Dewaraja, Y. K. \& Roberson, P. L. Methodology to incorporate biologically effective dose and equivalent uniform dose in patient-specific 3-dimensional dosimetry for non-Hodgkin lymphoma patients targeted with ${ }^{131}$ I-tositumomab therapy. J. Nucl. Med. 51, 654-659 (2010).

10. Fowler, J. F. Radiobiological aspects of low-dose rates in radioimmunotherapy. Int. J. Radiat. Oncol. Biol. Phys. 18, 1261-1269 (1990) Radiobiological treatment of RPT.

11. McDevitt, M. R. et al. Radioimmunotherapy with alpha-emitting nuclides. Eur. J. Nucl. Med. 25 , 1341-1351 (1998).

12. Wessels, B. W. \& Rogus, R. D. Radionuclide selection and model absorbed dose calculations for radiolabeled tumor associated antibodies. Med. Phys. 11, 638-645 (1984).

13. Bloomer, W. D., McLaughlin, W. H., Adelstein, S. J. $\&$ Wolf, A. P. Therapeutic applications of Auger and alpha emitting radionuclides. Strahlentherapie $\mathbf{1 6 0}$ 755-757 (1984)
14. O'Donoghue, J. A., Bardies, M. \& Wheldon, T. E. Relationships between tumor size and curability for uniformly targeted therapy with beta-emitting radionuclides. J. Nucl. Med. 36, 1902-1909 (1995). Demonstrates that, in contrast to external-beam radiotherapy, in RPT fewer cells do not lead to greater tumour control probability.

15. Cherry, S. R., Sorenson, J. A., \& Phelps, M. E. Physics in Nuclear Medicine e-Book (Elsevier Health Sciences, 2012).

16. Behr, T. M. et al. Therapeutic advantages of Auger electron- over beta-emitting radiometals or radioiodine when conjugated to internalizing antibodies. Eur. J. Nucl. Med. 27, 753-765 (2000).

17. Bodei, L., Kassis, A. I., Adelstein, S. J \& Mariani, G. Radionuclide therapy with iodine-125 and other Auger-electron-emitting radionuclides: experimental models and clinical applications. Cancer Biother. Radiopharm. 18, 861-877 (2003).

18. Howell, R. W. et al. in Biophysical Aspects of Auger Processes (Howell, R. W., Narra, V. R., Sastry, K. S. R. \& Rao, D. V. Eds). 290-318 (Medical Physics, 1991).

19. Kassis, A. I., Adelstein, S. J. \& Mariani, G. Radiolabeled nucleoside analogs in cancer diagnosis and therapy. Q. J. Nucl. Med. 40, 301-319 (1996). 
20. Kiess, A. P. et al. Auger radiopharmaceutical therapy targeting prostate-specific membrane antigen. J. Nucl. Med. 56, 1401-1407 (2015)

21. Macapinlac, H. A. et al. Pilot clinical trial of 5 -[125I]iodo-2'-deoxyuridine in the treatment of colorectal cancer metastatic to the liver. J. Nucl. Med. 37 (Suppl. 4), 25-29 (1996).

22. Daghighian, F. et al. Pharmacokinetics and dosimetry of iodine-125-IUdR in the treatment of colorectal cancer metastatic to liver. J. Nucl. Med. 37 (Suppl. 4), 29-32 (1996)

23. Sgouros, G. et al. Mathematical model of $5-[125]]$ iodo-2'-deoxyuridine treatment: continuous infusion regimens for hepatic metastases. Int. J. Radiat. Oncol. Biol. Phys. 41, 1177-1183 (1998).

24. Rebischung, $C$. et al. First human treatment of resistant neoplastic meningitis by intrathecal administration of MTX plus ${ }^{125} \mathrm{JUdR}$. Int. J. Radiat. Biol. 84, 1123-1129 (2008)

25. Behr, T. M. et al. Therapeutic efficacy and dose-limiting toxicity of Auger-electron vs. beta emitters in radioimmunotherapy with internalizing antibodies: evaluation of ${ }^{125} \mathrm{I}$ - vs. ${ }^{131} \mathrm{I}$-labeled $\mathrm{CO} 17-1 \mathrm{~A}$ in a human colorectal cancer model. Int. J. Cancer. 76, 738-748 (1998).

26. Ku, A., Facca, V. J., Cai, Z. \& Reilly, R. M. Auger electrons for cancer therapy - a review. EJNMM Radiopharm. Chem. 4, 27 (2019).

27. Brooks, R. C. et al. Metal complexes of bleomycin evaluation of [Rh-105]-bleomycin for use in targeted radiotherapy. Nucl Med. Biol. 26, 421-430 (1999).

28. Miao, Y., Owen, N. K., Fisher, D. R., Hoffman, T. J. \& Quinn, T. P. Therapeutic efficacy of a ${ }^{188}$ Re-labeled alpha-melanocyte-stimulating hormone peptide analog in murine and human melanoma-bearing mouse models. J. Nucl. Med. 46, 121-129 (2005).

29. Champion, C., Quinto, M. A., Morgat, C. Zanotti-Fregonara, P. \& Hindiē, E. Comparison between three promising $\beta$-emitting radionuclides, ${ }^{67} \mathrm{Cu},{ }^{47} \mathrm{Sc}$ and ${ }^{161} \mathrm{~Tb}$, with emphasis on doses delivered to minimal residual disease. Theranostics 6, 1611-1618 (2016)

30. Howell, R. W., Goddu, S. M. \& Rao, D. V. Application of the linear-quadratic model to radioimmunotherapy: further support for the advantage of longer-lived radionuclides. J. Nucl. Med. 35, 1861-1869 (1994).

31. Watson, E. E., Stabin, M. G., Davis, J. L. \& Eckerman, K. F. A model of the peritoneal cavity for use in internal dosimetry. J. Nucl. Med. 30, 2002-2011 (1989)

32. Zimmermann, R. G. Why are investors not interested in my radiotracer? The industrial and regulatory constraints in the development of radiopharmaceuticals. Nucl. Med. Biol. 40, 155-166 (2013).

33. Mikheev, N. B. Radioactive colloidal solutions and suspensions for medical use. At. Energy Rev. 14 3-36 (1976)

34. Bayly, R. J., Peacegood, J. A. \& Peake, S. C. ${ }^{90}$ Y ferric hydroxide colloid. Ann. Rheum. Dis. 32 (Suppl.), 10 (1973).

35. Washburn, L. C. et al. ${ }^{90}$ Y-labeled monoclonal antibodies for cancer therapy. Int. J. Radiat. Appl. Instrum Part B Nucl. Med Biol. 13, 453-456 (1986)

36. Kozak, R. W. et al. Nature of the bifunctional chelating agent used for radioimmunotherapy with yttrium-90 monoclonal antibodies: critical factors in determining in vivo survival and organ toxicity. Cancer Res. 49 2639-2644 (1989).

37. Brechbiel, M. W. \& Gansow, O. A. Backbonesubstituted DTPA ligands for ${ }^{90} \mathrm{Y}$ radioimmunotherapy. Bioconjug. Chem. 2, 187-194 (1991). Introduces a novel DTPA chelate for radioimmunotherapy with metallic radionuclides.

38. Stewart, J. S. et al. Intraperitoneal radioimmunotherapy for ovarian cancer: pharmacokinetics, toxicity, and efficacy of I-131 labeled monoclonal antibodies. Int. J. Radiat. Oncol. Biol. Phys. 16, 405-413 (1989).

39. Oei, A. L. et al. Decreased intraperitoneal disease recurrence in epithelial ovarian cancer patients receiving intraperitoneal consolidation treatment with yttrium-90-labeled murine HMFG 1 without improvement in overall survival. Int. J. Cancer 120 2710-2714 (2007)

40. Waldmann, T. A. et al. Radioimmunotherapy of interleukin-2R alpha-expressing adult T-cell leukemia with yttrium-90-labeled anti-Tac. Blood 86, 4063-4075 (1995)

41. Foss, F. M. et al. Phase I study of the pharmacokinetics of a radioimmunoconjugate, ${ }^{90} \mathrm{Y}-\mathrm{T} 101$, in patients with CD5-expressing leukemia and lymphoma. Clin. Cancer Res. 4, 2691-2700 (1998).
42. Witzig, T. E. et al. Phase I/II trial of IDEC-Y2B8 radioimmunotherapy for treatment of relapsed or refractory $\mathrm{CD} 20^{+}$B-cell non-Hodgkin's lymphoma. J. Clin. Oncol. 17, 3793-3803 (1999).

43. Nisa, L., Savelli, G. \& Giubbini, R. Yttrium-90 DOTATOC therapy in GEP-NET and other SST2 expressing tumors: a selected review. Ann. Nucl. Med. 25, 75-85 (2011)

44. Salem, R. \& Thurston, K. G. Radioembolization with ${ }^{90}$ yttrium microspheres: a state-of-the-art brachytherapy treatment for primary and secondary liver malignancies. Part 1: technical and methodologic considerations. J. Vasc. Interv. Radiol. 17, 1251-1278 (2006)

45. Lau, W. Y. et al. Selective internal radiation therapy for nonresectable hepatocellular carcinoma with intraarterial infusion of ${ }^{90} \mathrm{yttrium}$ microspheres. Int. J. Radiat. Oncol. Biol. Phys. 40, 583-592 (1998). Early report of microsphere therapy for hepatic artery infusion

46. Popperl, G. et al. Selective internal radiation therapy with SIR-Spheres in patients with nonresectable live tumors. Cancer Biother. Radiopharm. 20, 200-208 (2005).

47. Mancini, R. et al. A multicentric phase Il clinical tria on intra-arterial hepatic radiotherapy with ${ }^{90}$ yttrium SIR-spheres in unresectable, colorectal liver metastases refractory to i.v. chemotherapy: preliminary results on toxicity and response rates. In Vivo 20, 711-714 (2006)

48. Maleux, G. et al. Yttrium-90 radioembolization for the treatment of chemorefractory colorectal liver metastases: technical results, clinical outcome and factors potentially influencing survival. Acta Oncol. $\mathbf{5 5}$ 486-495 (2016)

49. Yue, J. et al. Comparison of quantitative Y-90 SPECT and non-time-of-flight PET imaging in post-therap radioembolization of liver cancer. Med. Phys. $\mathbf{4 3}$ 5779 (2016)

50. Das, T. $\&$ Banerjee, S. Theranostic applications of lutetium-177 in radionuclide therapy. Curr. Radiopharm. 9, 94-101 (2016).

51. Tarasov, V. A., Andreev, O. I., Romanov, E. G. Kuznetsov, R. A., Kupriyanov, V. V. \& Tselishchev, I. V. Production of no-carrier added lutetium-177 by irradiation of enriched ytterbium-176. Curr. Radiopharm. 8, 95-106 (2015).

52. Banerjee, S., Pillai, M. R. A. \& Knapp, F. F. Lutetium-177 therapeutic radiopharmaceuticals: linking chemistry, radiochemistry, and practical applications. Chem. Rev. 115, 2934-2974 (2015).

53. Sgouros, G. et al. MIRD Monograph: Radiobiology and Dosimetry for Radiopahrmaceutical Therapy with Alpha-Particle Emitters (ed. Sgouros, G.). (SNMMI, 2015)

Comprehensive review of radiobiology and dosimetry for a-emitter RPT.

54. Sgouros, G. et al. MIRD pamphlet no. 22 (abridged) radiobiology and dosimetry of alpha-particle emitters for targeted radionuclide therapy. J. Nucl. Med. $\mathbf{5 1}$ 311-328 (2010)

55. Parker, C. et al. Targeted alpha therapy, an emerging class of cancer agents a review. JAMA Oncol. 4 , 1765-1772 (2018).

56. Parker, C. et al. Alpha emitter radium-223 and survival in metastatic prostate cancer. N. Engl. J. Med. 369, 213-223 (2013).

ASYMPCA trial report that led to approval of radium-223 for treatment of patients with prostate cancer with bone metastases.

57. Kratochwil, C. et al. ${ }^{225}$ Ac-PSMA-617 for PSMA-targeted $\alpha$-radiation therapy of metastatic castration-resistant prostate cancer. J. Nucl. Med. 57, 1941-1944 (2016) Striking imaging-based demonstration of the remarkable response that may be obtained with $\alpha$-emitter RPT.

58. Kratochwil, C., Haberkorn, U. \& Giesel, F. L. Radionuclide therapy of metastatic prostate cancer. Semin. Nucl. Med. 49, 313-325 (2019).

59. Parker, C., Heidenreich, A., Nilsson, S. \& Shore, N. Current approaches to incorporation of radium-223 in clinical practice. Prostate Cancer Prostatic Dis. $\mathbf{2 1}$ 37-47 (2018)

60. Subbiah, V. et al. Alpha particle radium 223 dichloride in high-risk osteosarcoma: a phase I dose escalation trial. Clin. Cancer Res. 25, 3802-3810 (2019).

61. Geva, R. et al, Radium-223 in combination with paclitaxel in cancer patients with bone metastases: safety results from an open-label, multicenter phase Ib study. Eur. J. Nucl. Med. Mol. Imaging 46 , 1092-1101 (2019).
62. Takalkar, A., Paryani, B., Adams, S. \& Subbiah, V. Radium-223 dichloride therapy in breast cancer with osseous metastases. BMJ Case Rep. 2015, bcr2015211152 (2015)

63. Eckerman, K. F. \& Enzo, A. MIRD: Radionuclide Data and Decay Schemes, 2nd edn. (Society of Nuclear Medicine, 2008)

64. Hellman, S., Devita V. T. and Rosenberg, S. A Cancer: Principles \& Practice of Oncology. 265-288 (Lippincott Williams \& Wilkins, 2001).

65. Longcor, J. \& Oliver, K. Phase 1, open-label, dose escalation study of I-131-CLR1404 (CLR 131) in patients with relapsed or refractory multiple myeloma. Blood 134, 1864 (2019)

66. Ciernik, I. F. et al. Proton-based radiotherapy for unresectable or incompletely resected osteosarcoma. Cancer 117, 4522-4530 (2011).

67. Oertel, S. et al. Radiotherapy in the treatment of primary osteosarcoma-a single center experience. Tumori 96, 582-588 (2010).

68. Senthamizhchelvan, S et al. Tumor dosimetry and response for ${ }^{153} \mathrm{Sm}$-ethylenediamine tetramethylene phosphonic acid therapy of high-risk osteosarcoma. J. Nucl. Med. 53, 215-224 (2012)

69. Hobbs, R. F. et al. A treatment planning method for sequentially combining radiopharmaceutical therapy and external radiation therapy. Int. J. Radiat. Oncol. Biol. Phys. 80, 1256-1262 (2011)

70. Wessels, B. W. et al. MIRD pamphlet no. 20: the effect of model assumptions on kidney dosimetry and response-implications for radionuclide therapy. J. Nucl. Med. 49, 1884-1899 (2008).

71. Kiess, A. P. et al. (2S)-2-(3-(1-Carboxy-5-(4-211 At-astatobenzamido)pentyl)ureido)-pentanedioic acid for PSMA-targeted alpha-particle radiopharmaceutical therapy. J. Nucl. Med. 57, 1569-1575 (2016).

72. Jentzen, W. et al. Pre-therapeutic I-124 PET(/CT) dosimetry confirms low average absorbed doses per administered $\mathrm{I}-131$ activity to the salivary glands in radioiodine therapy of differentiated thyroid cancer. Eur. J. Nucl. Med. Mol. Imaging 37, 884-895 (2010).

73. Hobbs, R. F., Jentzen, W., Bockisch, A. \& Sgouros, G. Monte Carlo-based 3-dimensional dosimetry of salivary glands in radioiodine treatment of differentiated thyroid cancer estimated using ${ }^{124}$ I PET. O. J. Nucl. Med. Mol. Imaging 57, 79-91 (2013).

74. Hobbs, R. F. et al. A bone marrow toxicity model for ${ }^{223} \mathrm{Ra}$ alpha-emitter radiopharmaceutical therapy. Phys. Med. Biol. 57, 3207-3222 (2012).

75. Back, T. \& Jacobsson, L. The alpha-camera: a quantitative digital autoradiography technique using a charge-coupled device for ex vivo highresolution bioimaging of alpha-particles. J. Nucl. Med. 51, 1616-1623 (2010).

a-Camera imaging technique to assess distribution of $\alpha$-particles in tissues.

76. Miller, B. W. et al. Quantitative single-particle digital autoradiography with alpha-particle emitters for targeted radionuclide therapy using the iQID camera. Med. Phys. 42, 4094-4105 (2015).

77. Miller, B. W. Radiation imagers for quantitative single-particle digital autoradiography of alpha- and beta-particle emitters. Semin. Nucl. Med. $\mathbf{4 8}$ 367-376 (2018)

78. Ljungberg, M. \& Gleisner, K. S. 3-D image-based dosimetry in radionuclide therapy. IEEE Trans. Radiat Plasma Med. Sci. 2, 527-540 (2018).

79. Sgouros, G. \& Hobbs, R. F. Dosimetry for radiopharmaceutical therapy. Semin. Nucl. Med. 44 , 172-178 (2014)

80. Dewaraja, Y. K., Ljungberg, M., Green, A. J., Zanzonico, P. B. \& Frey, E. C. MIRD pamphlet no. 24: guidelines for quantitative ${ }^{131}$ I SPECT in dosimetry applications. J. Nucl. Med. 54, 122390 (2013).

81. Bolch, W. E., Eckerman, K. F., Sgouros, G. \& Thomas, S. R. MIRD pamphlet no. 21: a generalized schema for radiopharmaceutical dosimetrystandardization of nomenclature. J. Nucl. Med. $\mathbf{5 0}$ 477-484 (2009).

Mathematical formalism for radiopharmaceutical dosimetry.

82. Vaziri, B., Wu, H., Dhawan, A. P., Du, P. \& Howell, R. W. MIRD pamphlet no. 25: MIRDcell V2.0 software tool for dosimetric analysis of biologic respons of multicellular populations. J. Nucl. Med. 55 1557-1564 (2014).

83. Dewaraja, Y. K. et al. MIRD pamphlet no. 23 quantitative SPECT for patient-specific 3-dimensional dosimetry in internal radionuclide therapy. J. Nucl. Med. 53, 1310-1325 (2012). 
84. Bolch, W. E. et al. MIRD pamphlet no. 17: the dosimetry of nonuniform activity distributionsradionuclide $\mathrm{S}$ values at the voxel level. Medical Internal Radiation Dose Committee. J. Nucl. Med. 40, 11S-36S (1999).

85. Furhang, E. E., Chui, C. S., Kolbert, K. S., Larson, S. M \& Sgouros, G. Implementation of a Monte Carlo dosimetry method for patient-specific internal emitter therapy. Med. Phys. 24, 1163-1172 (1997).

86. Kolbert, K. S. et al. Implementation and evaluation of patient-specific three-dimensional internal dosimetry. J. Nucl. Med. 38, 301-308 (1997).

87. Cremonesi, M. et al. Correlation of dose with toxicity and tumour response to Y-90- and Lu-177-PRRT provides the basis for optimization through individualized treatment planning. Eur. J. Nucl. Med. Mol. Imaging 45, 2426-2441 (2018).

88. Pacilio, M. et al. A case report of image-based dosimetry of bone metastases with Alpharadin (Ra-223-dichloride) therapy: inter-fraction variability of absorbed dose and follow-up. Ann. Nucl. Med. 30 , 163-168 (2016)

89. Stokke, C. et al. Dosimetry-based treatment planning for molecular radiotherapy: a summary of the 2017 report from the Internal Dosimetry Task Force. EJNMMI Phys. 4, 27 (2017).

90. Dewaraja, Y. K. et al. Tumor-absorbed dose predicts progression-free survival following ${ }^{131}$ I-tositumomab radioimmunotherapy. J. Nucl. Med. 55, 1047-1053 (2014).

91. O'Donoghue, J. A. et al. Hematologic toxicity in radioimmunotherapy: dose-response relationships for I-131 labeled antibody therapy. Cancer Biother. Radiopharm. 17, 435-443 (2002)

92. Sgouros, G. \& Goldenberg, D. M. Radiopharmaceutical therapy in the era of precision medicine. Eur. J. Cancer 50, 2360-2363 (2014).

93. Hertz, S. \& Roberts, A. Radioactive iodine in the study of thyroid physiology. 7 . The use of radioactive iodine therapy in hyperthyroidism. JAMA 131, 81-86 (1946).

\section{Potential of radioiodine to treat thyroid disease.}

94. Campbell, J. E., Robajdek, E. S. \& Anthony, D. S. The metabolism of Ac-227 and its daughters Th-227 and Ra-223 by rats. Radiat. Res. 4, 294-302 (1956)

95. Joshi, D. P., Seery, W. H., Goldberg, L. G. \& Goldman, L. Evaluation of phosphorus 32 for intractable pain secondary to prostatic carcinoma metastasES. JAMA 193, 621-623 (1965).

96. Harrison, G. E., Carr, T. E., Sutton, A. \& Rundo, J. Plasma concentration and excretion of calcium-47, strontium-85, barium-133 and radium-223 following successive intravenous doses to a healthy man. Nature 209, 526-527 (1966).

97. Foss, C. A. et al. Radiolabeled small-molecule ligands for prostate-specific membrane antigen: in vivo imaging in experimental models of prostate cancer. Clin. Cancer Res. 11, 4022-4028 (2005)

98. Zechmann, C. M. et al. Radiation dosimetry and first therapy results with a ${ }^{124} \mathrm{I} / 131 \mathrm{I}$-labeled small molecule (MIP-1095) targeting PSMA for prostate cancer therapy. Eur. J. Nucl. Med. Mol. Imaging 41 1280-1292 (2014).

99. Kong, G. \& Hicks, R. J. Peptide receptor radiotherapy: current approaches and future directions. Curr. Treat. Options Oncol. 20, 77 (2019).

100. Muller, C., Vlahov, I. R., Santhapuram, H. K. R. Leamon, C. P. \& Schibli, R. Tumor targeting using Ga-67-DOTA-Bz-folate - investigations of methods to improve the tissue distribution of radiofolates. Nucl. Med. Biol. 38, 715-723 (2011).

101. Muller, C., Struthers, H., Winiger, C., Zhernosekov, K. $\&$ Schibli, R. DOTA conjugate with an albumin-binding entity enables the first folic acid-targeted ${ }^{177} \mathrm{Lu}$ radionuclide tumor therapy in mice. J. NuCl. Med. $\mathbf{5 4}$, 124-131 (2013)

102. Ambrosini, V., Fani, M., Fanti, S., Forrer, F \& Maecke, H. R. Radiopeptide imaging and therapy in Europe. J. Nucl. Med. 52, 42S-55S (2011).

103. Song, H. \& Sgouros, G. Radioimmunotherapy of solid tumors: searching for the right target. Curr. Drug Deliv. 8, 26-44 (2011).

104. Leaman Alcibar, O. et al. Time for radioimmunotherapy: an overview to bring improvements in clinical practice. Clin. Trans/ Oncol. 21, 992-1004 (2019)

105. Larson, S. M., Carrasquillo, J. A. \& Reynolds, J. C. Radioimmunodetection and radioimmunotherapy. Cancer Invest. 2, 363-381 (1984).

106. Goldenberg, D. M. Targeting of cancer with radiolabeled antibodies. Prospects for imaging and therapy. Arch. Pathol. Lab. Med. 112, 580-587 (1988).
107. Jeon, J. Review of therapeutic applications of radiolabeled functional nanomaterials. Int. J. Mol. Sci 20, 2323 (2019)

108. Sofou, S. \& Sgouros, G. Antibody-targeted liposomes in cancer therapy and imaging. Expert. Opin. Drug Deliv 5, 189-204 (2008).

109. Lee, E. J., Chung, H. W., Jo, J. H. \& So, Y. Radioembolization for the treatment of primary and metastatic liver cancers. Nucl. Med. Mol. Imaging 53 367-373 (2019)

110. Wu, A. M. \& Senter, P. D. Arming antibodies: prospects and challenges for immunoconjugates. Nat. Biotech. 23, 1137-1146 (2005).

111. Hu, S. Z. et al. Minibody: a novel engineered anti-carcinoembryonic antigen antibody fragment (single-chain $\mathrm{Fv}^{-} \mathrm{C}_{\mathrm{H}} 3$ ) which exhibits rapid, high-level targeting of xenografts. Cancer Res. 56, 3055-3061 (1996).

112. Wu, A. M. et al. Tumor localization of anti-CEA single-chain Fvs: improved targeting by non-covalent dimers. Immunotechnology 2, 21-36 (1996).

113. Cheung, N. K. V. et al. Single-chain Fv-streptavidin substantially improved therapeutic index in multistep targeting directed at disialoganglioside GD2. J. Nucl. Med. 45, 867-877 (2004).

114. Boerman, O. C. et al. Pretargeting of renal cell carcinoma: improved tumor targeting with a bivalent chelate. Cancer Res. 59, 4400-4405 (1999).

115. Chang, C. H. et al. Molecular advances in pretargeting radioimunotherapy with bispecific antibodies. Mol. Cancer Ther. 1, 553-563 (2002).

116. McBride, W. J. et al. Bispecific antibody pretargeting PET (ImmunoPET) with an I-124-labeled haptenpeptide. J. Nucl. Med. 47, 1678-1688 (2006)

117. Catz, B. et al. Treatment of cancer of the thyroid postoperatively with suppressive thyroid medication, radioactive iodine, and thyroid-stimulating hormone. Cancer 12, 371-383 (1959).

118. Hamilton, J. G. \& Soley, M. H. Studies in iodine metabolism of the thyroid gland in situ by the use of radio-iodine in normal subjects and in patients with various types of goiter. Am. J. Physiol. 131, 0135-0143 (1940).

119. Benua, R. S., Rawson, R. W., Sonenberg, M. \& Cicale, N. R. Relation of radioiodine dosimetry to results and complications in treatment of metastatic thyroid cancer. Am. J. Roentgenol. Radium Ther. Nucl. Med. 87, 171-182 (1962).

120. Maxon, H. R. et al. Relation between effective radiation dose and outcome of radioiodine therapy for thyroid cancer. N. Engl. J. Med. 309, 937-941 (1983).

121. Livingood, J. J. \& Seaborg, G. T. Radioactive iodine isotopes. Phys. Rev. 53, 1015 (1938)

122. Knapp, R. F. F. \& Dash, A. Radiopharmaceuticals for Therapy - 2016. (Springer, 2016).

123. Hertz, S., Roberts, A., Means, J. H. \& Evans, R. D. Radioactive iodine as an indicator in thyroid physiology - lodine collection by normal and hyperplastic thyroids in rabbits. Am. J. Physiol. 128, 565-576 (1940).

124. Cooper, D. S. et al. Revised American Thyroid Association management guidelines for patients with thyroid nodules and differentiated thyroid cancer. Thyroid 19, 1167-1214 (2009).

125. Fagin, J. A. \& Wells, S. A. Jr. Biologic and clinical perspectives on thyroid cancer. N. Engl. J. Med. 375 , 1054-1067 (2016).

126. Gao, W. et al. Internal radiotherapy using ${ }^{32} \mathrm{P}$ colloid or microsphere for refractory solid tumors. Ann. Nucl. Med. 22, 653-660 (2008).

127. Morris, M. J. et al. Radium-223 mechanism of action: implications for use in treatment combinations. Nat. Rev. Urol. 16, 745-756 (2019).

128. Leung, C. N. et al. Dose-dependent growth delay of breast cancer xenografts in the bone marrow of mice treated with ${ }^{223} \mathrm{Ra}$ : the role of bystander effects and their potential for therapy. J. Nucl. Med. 61, 89-95 (2020).

129. Tombal, B. F. et al. Decreased fracture rate by mandating bone-protecting agents in the EORTC 1333/PEACE III trial comparing enzalutamide and Ra223 versus enzalutamide alone: an interim safety analysis. J. Clin. Oncol. 37, 5007 (2019).

130. Smith, M. R. et al. ERA 223: a phase 3 trial of radium-223 dichloride (Ra-223) in combination with abiraterone acetate (abiraterone) and prednisone in the treatment of asymptomatic or mildly symptomatic chemotherapy-naïve patients (pts) with bone predominant metastatic castration-resistant prostate cancer (mCRPC). J. Clin. Oncol. 33, TPS5082 (2015).
131. Dalla Volta, A., Formenti, A. M. \& Berruti, A. Higher risk of fragility fractures in prostate cancer patients treated with combined radium-223 and abiraterone: prednisone may be the culprit. Eur. Urol. 75 894-895 (2019).

132. Sartor, O. Overview of samarium Sm 153 lexidronam in the treatment of painful metastatic bone disease. Rev. Urol. 6, S3-S12 (2004).

133. Anderson, P. M., Subbiah, V. \& Rohren, E. In: Current Advances in Osteosarcoma. 291-304 (Springer International Publishing, 2014).

134. Longo, J., Lutz, S. \& Johnstone, C. Samarium153-ethylene diamine tetramethylene phosphonate, a beta-emitting bone-targeted radiopharmaceutical, useful for patients with osteoblastic bone metastases. Cancer Manag. Res. 5, 235-242 (2013).

135. Chirby, D., Franck, S. \& Troutner, D. E. Adsorption of ${ }^{153} \mathrm{Sm}$-EDTMP on calcium hydroxyapatite. Int. $J$. Radiat. Appl. Instrum. Part A Appl. Radiat. Isotopes 39, 495-499 (1988)

136. Eary, J. F. et al. Samarium-153-EDTMP biodistribution and dosimetry estimation. J. Nucl. Med. 34 , 1031-1036 (1993).

137. Goyal, J. \& Antonarakis, E. S. Bone-targeting radiopharmaceuticals for the treatment of prostate cancer with bone metastases. Cancer Lett. 323, 135-146 (2012).

138. Simon, J. J. et al. Preclinical evaluation of Sm-153-DOTMP as a therapeutic bone-seeking radiopharmaceutical. J. Nucl. Med. 52, 1751 (2011).

139. Simón, J. et al. A preclinical investigation of the saturation and dosimetry of ${ }^{153} \mathrm{Sm}$-DOTMP as a bone-seeking radiopharmaceutical. Nucl. Med. Biol. 39, 770-776 (2012)

140. Schmidt, M., Hero, B. \& Simon, T. I-131-mIBG therapy in neuroblastoma: established role and prospective applications. Clin. Transl Imaging 4, 87-101 (2016).

141. Schoot, R. A et al. The role of ${ }^{131}$ Imetaiodobenzylguanidine (MIBG) therapy in unresectable and compromising localised neuroblastoma. Eur. J. Nucl. Med. Mol. Imaging 40, 1516-1522 (2013).

142. George, S. L. et al. Individualized I-131-mIBG therapy in the management of refractory and relapsed neuroblastoma. Nucl. Med. Commun. 37, 466-472 (2016)

143. Eisenhut, M. M. W. in Handbook of Nuclear Chemistry. (eds Vértes, A. N. S., Klencsár, Z., Lovas, R. G., Rösch, F.) (Springer, 2011).

144. Inaki, A. et al. A phase I clinical trial for [ $\left.{ }^{131} I\right]$ metaiodobenzylguanidine therapy in patients with refractory pheochromocytoma and paraganglioma: a study protocol. J. Med. Invest. 64, 205-209 (2017).

145. Modak, S. et al. Arsenic trioxide as a radiation sensitizer for ${ }^{131}$-metaiodobenzylguanidine therapy: results of a phase II study. J. Nucl. Med. 57, 231-237 (2016).

146. Shilkrut, M., Bar-Deroma, R., Bar-Sela, G. Berniger, A. \& Kuten, A. Low-dose iodine-131 metaiodobenzylguanidine therapy for patients with malignant pheochromocytoma and paraganglioma: single center experience. Am. J. Clin. Oncol. 33 , 79-82 (2010)

147. Coleman, R. E. et al. Radiation dosimetry, pharmacokinetics, and safety of ultratrace lobenguane l-131 in patients with malignant pheochromocytoma/ paraganglioma or metastatic carcinoid. Cancer Biother. Radiopharm 24, 469-475 (2009).

148. Gonias, S. et al. Phase II study of high-dose [ $\left.{ }^{131} I\right]$ metaiodobenzylguanidine therapy for patients with metastatic pheochromocytoma and paraganglioma. J. Clin. Oncol. 27, 4162-4168 (2009)

149. Fitzgerald, P. A. et al. Malignant pheochromocytomas and paragangliomas: a phase II study of therapy with high-dose ${ }^{131}$ I-metaiodobenzylguanidine ( ${ }^{131}$ I-MIBG) Ann. NY Acad. Sci. 1073, 465-490 (2006).

150. Sisson, J. C et al. Treatment of malignant pheochromocytomas with 131-I metaiodobenzylguanidine and chemotherapy. Am. J. Clin. Oncol. 22, 364-370 (1999).

151. Mundschenk, J., Kopf, D. \& Lehnert, H. Therapy of malignant pheochromocytoma. Invitation to participate in a randomized multicenter study. Dtsch. medizinische Wochenschr. 123, 32-33 (1998)

152. Noto, R. B. et al. Phase 1 study of high-specific-activity I-131 MIBG for metastatic and/or recurrent pheochromocytoma or paraganglioma. J. Clin. Endocrinol. Metab. 103, 213-220 (2017).

153. Pinto, J. T. et al. Prostate-specific membrane antigen: a novel folate hydrolase in human prostatic carcinoma cells. Clin. Cancer Res. 2, 1445-1451 (1996). 
154. Heston, W. D. Characterization and glutamyl preferring carboxypeptidase function of prostate specific membrane antigen: a novel folate hydrolase. Urology 49 (Suppl. 3A), 104-112 (1997).

155. Murphy, D. G., Sathianathen, N., Hofman, M. S., Azad, A. \& Lawrentschuk, N. Where to next for theranostics in prostate cancer? Eur. Urol. Oncol. 2 163-165 (2019)

156. Tateishi, U. Prostate-specific membrane antigen (PSMA)-ligand positron emission tomography and radioligand therapy (RLT) of prostate cancer. Jap. J Clin. Oncol. 50, 349-356 (2020).

157. Novakova, Z. et al. Design of composite inhibitors targeting glutamate carboxypeptidase II: the importance of effector functionalities. FEBS J. 283, 130-143 (2016).

158. Banerjee, S. R. et al. 68Ga-labeled inhibitors of prostate-specific membrane antigen (PSMA) for imaging prostate cancer. J. Med. Chem. 53 5333-5341 (2010)

159. Barinka, C. et al. Structural insight into the pharmacophore pocket of human glutamate carboxypeptidase II. J. Med. Chem. 50, 3267-3273 (2007).

160. Bařinka, C., Rojas, C., Slusher, B. \& Pomper, M Glutamate carboxypeptidase II in diagnosis and treatment of neurologic disorders and prostate cancer. Curr. Med. Chem. 19, 856-870 (2012).

161. Kozikowski, A. P. et al. Synthesis of urea-based inhibitors as active site probes of glutamate carboxypeptidase II: efficacy as analgesic agents. J. Med. Chem. 47, 1729-1738 (2004). Early report on small-molecule PSMA inhibitors.

162. Zhou, J., Neale, J. H., Pomper, M. G. \& Kozikowski, A. P. NAAG peptidase inhibitors and their potential for diagnosis and therapy. Nat. Rev. Drug Discov. 4, 1015-1026 (2005)

163. Wu, L. Y. et al. The molecular pruning of a phosphoramidate peptidomimetic inhibitor of prostate-specific membrane antigen. Bioorg. Med Chem. 15, 7434-7443 (2007)

164. Liu, T., Toriyabe, Y., Kazak, M. \& Berkman, C. E. Pseudoirreversible inhibition of prostate-specific membrane antigen by phosphoramidate peptidomimetics. Biochemistry 47, 12658-12660 (2008).

165. Choy, C. J. et al. Lu-177-labeled phosphoramidatebased PSMA inhibitors: the effect of an albumin binder on biodistribution and therapeutic efficacy in prostate tumor-bearing mice. Theranostics 7 1928-1939 (2017).

166. Hofman, M. S. et al. [ $\left.{ }^{177} \mathrm{Lu}\right]-P S M A-617$ radionuclide treatment in patients with metastatic castrationresistant prostate cancer (LuPSMA trial): a singlecentre, single-arm, phase 2 study. Lancet Oncol. 19 825-833 (2018)

Efficacy and toxicity of anti-PSMA therapy in prostate cancer using lutetium-177.

167. Derlin, T. \& Schmuck, S. [177Lu]-PSMA-617 radionuclide therapy in patients with metastatic castration-resistan prostate cancer. Lancet Oncol. 19, e372 (2018).

168. Rahbar, K., Ahmadzadehfar, H., Seifert, R. \& Boegemann, M. ${ }^{177}$ Lu]-PSMA-617 radionuclide therapy in patients with metastatic castration-resistant prostate cancer. Lancet Oncol. 19, e371 (2018).

169. Hofman, M. S., Violet, J., Hicks, R. J. \& Sandhu, S. [177 Lu]-PSMA-617 radionuclide therapy in patients with metastatic castration-resistant prostate cancer Author's reply. Lancet Oncol. 19, e373 (2018).

170. Muzio, V. et al. Assessment of in vivo biodistribution and treatment efficacy of Lu-177 PSMA-R2 and Lu-177-PSMA-617 on mice bearing prostate cancer tumors. Eur. J. Nucl. Med. Mol. Imaging 46 (Suppl. 1), 17 (2019).

171. Nedrow-Byers, J. R. et al. A phosphoramidate-based prostate-specific membrane antigen-targeted SPECT agent. Prostate 72, 904-912 (2012).

172. Choy, C. J. et al. ${ }^{177}$ Lu-labeled phosphoramidate-based PSMA inhibitors: the effect of an Albumin Binder on biodistribution and therapeutic efficacy in prostate tumor-bearing mice. Theranostics 7, 1928-1939 (2017).

173. Gourni, E. \& Henriksen, G. Metal-based PSMA radioligands. Molecules 22, 523 (2017).

174. Wester, H.-J. \& Schottelius, M. PSMA-targeted radiopharmaceuticals for imaging and therapy. Semin. Nucl. Med. 49, 302-312 (2019).

175. Ross, J. F., Chaudhuri, P. K. \& Ratnam, M. Differential regulation of folate receptor isoforms in normal and malignant tissues in vivo and in established cell lines. Physiologic and Clinical Implications. Cancer 73, 2432-2443 (1994).
176. Cheung, A. et al. Targeting folate receptor alpha for cancer treatment. Oncotarget. 7, 52553-52574 (2016).

177. Kettenbach, K. et al. Comparison study of two differently clicked ${ }^{18} \mathrm{~F}$-folates-lipophilicity plays a key role. Pharmaceuticals 11, 30 (2018).

178. Siwowska, K. \& Müller, C. Preclinical development of small-molecular-weight folate-based radioconjugates: a pharmacological perspective. O. J. Nucl. Med. Mol. Imaging 59, 269-286 (2015).

179. Siwowska, K. et al. Therapeutic potential of ${ }^{47} \mathrm{Sc}$ in comparison to ${ }^{177} \mathrm{Lu}$ and ${ }^{90} \mathrm{Y}$ : preclinical investigations. Pharmaceutics 11, 424 (2019).

180. Gupta, A. et al. Voxel-based dosimetry of iron oxide nanoparticle-conjugated ${ }^{177} \mathrm{Lu}$-labeled folic acid using SPECT/CT imaging of mice. Mol. Pharmaceutics. 16 1498-1506 (2019).

181. Müller, C. et al. Direct in vitro and in vivo comparison of ${ }^{161} \mathrm{~Tb}$ and ${ }^{177} \mathrm{Lu}$ using a tumour-targeting folate conjugate. Eur. J. Nucl. Med. Mol. Imaging 41 476-485 (2014).

182. Snyder, F. \& Wood, R. Alkyl and alk-1-enyl ethers of glycerol in lipids from normal and neoplastic human tissues. Cancer Res. 29, 251-257 (1969).

183. Snyder, F., Blank, M. L. \& Morris, H. P. Occurrence and nature of O-alkyl and O-alk-I-enyl moieties of glycerol in lipids of Morris transplanted hepatomas and normal rat liver. Biochim. Biophys. Acta 176 502-510 (1969)

184. Counsell, R. E., Schwendner, S. W., Meyer, K. L. Haradahira, T. \& Gross, M. D. Tumor visualization with a radioiodinated phospholipid ether. J. Nucl. Med. 31 332-336 (1990)

185. Meyer, K. L., Schwendner, S. W. \& Counsell, R. E. Potential tumor or organ-imaging agents. 30 Radioiodinated phospholipid ethers. J. Med. Chem. 32, 2142-2147 (1989).

186. Pinchuk, A. N. et al. Synthesis and structure-activity relationship effects on the tumor avidity of radioiodinated phospholipid ether analogues. J. Med. Chem. 49, 2155-2165 (2006).

187. Weichert, J. P. et al. Alkylphosphocholine analogs for broad-spectrum cancer imaging and therapy. Sci. Transl Med. 6, 240 ra75 (2014) Phosphocholine-based RPT

188. Baiu, D. C. et al. Targeted molecular radiotherapy of pediatric solid tumors using a radioiodinated alkyl-phospholipid ether analog. J. Nucl. Med. 59, 244-250 (2018)

189. Hall, L. T. et al. PET/CT imaging of the diapeutic alkylphosphocholine analog I-124-CLR1404 in high and low-grade brain tumors. Am. J. NuCl. Med. Mol. Imaging 7, 157-166 (2017)

190. Hall, L. T. et al. I-124 CLR1404 PET/CT in high-grade primary and metastatic brain tumors. Mol. Imaging Biol. 22, 434-443 (2020)

191. Morris, Z S. et al. Therapeutic combination of radiolabeled CLR 1404 with external beam radiation in head and neck cancer model systems. Radiother. Oncol. 116, 504-509 (2015).

192. Bodei, L., Kwekkeboom, D. J., Kidd, M., Modlin, I. M. \& Krenning, E. P. Radiolabeled somatostatin analogue therapy of gastroenteropancreatic cancer. Semin. Nucl. Med. 46, 225-238 (2016).

193. Fani, M., Nicolas, G. P. \& Wild, D. Somatostatin receptor antagonists for imaging and therapy. J. Nucl. Med. 58, 61S-66S (2017). Trial that demonstrates the greater tumour uptake and absorbed dose with somatostatin receptor antagonists compared with agonists.

194. Strosberg, J. et al. Phase 3 trial of Lu-177-dotatate fo midgut neuroendocrine tumors. N. Engl. J. Med. 376 125-135 (2017).

195. Strosberg, J. et al. Health-related quality of life in patients with progressive midgut neuroendocrine tumors treated with ${ }^{177} \mathrm{Lu}$-Dotatate in the phase III NETTER-1 trial. J. Clin. Oncol. 36, 2578-2584 (2018). Trial that led to FDA approval of ${ }^{177}$ Lu-DOTATATE.

196. Kwekkeboom, D. J. et al. Treatment with the radiolabeled somatostatin analog $\left[{ }^{177} \mathrm{Lu}-\mathrm{DOTA}^{0}, \mathrm{Tyr}^{3}\right]$ octreotate: toxicity, efficacy, and survival. J. Clin. Oncol. 26, 2124-2130 (2008).

197. Atcher, R. W., Friedman, A. M. \& Hines, J. J. An improved generator for the production of $\mathrm{Pb}-212$ and Bi-212 from Ra-224. Appl. Radiat. Isot. 39, 283-286 (1988).

198. Delpassand, E. et al. First clinical experience using targeted alpha-emitter therapy with Pb-212DOTAMTATE (AlphaMedix ${ }^{\mathrm{TM}}$ ) in patients with SSTR(+) neuroendocrine tumors. J. Nucl. Med. 60, (2019).
199. Stallons, T. A. R., Saidi, A., Tworowska, I., Delpassand, E S. \& Torgue J J Preclinica Investigation of $\mathrm{Pb}$-212-DOTAMTATE for peptide receptor radionuclide therapy in a neuroendocrine tumor model. Mol. Cancer Ther 18, 1012-1021 (2019).

200. Bodei, L. et al. Long-term tolerability of PRRT in 807 patients with neuroendocrine tumours: the value and limitations of clinical factors. Eur. J. Nucl. Med. Mol. Imaging 42, 5-19 (2015)

Study that demonstrates the toxicity profile of PRRT with ${ }^{90} \mathrm{Y},{ }^{177} \mathrm{Lu}$ or their combination in a large series of patients

201. Reidy-Lagunes, D. et al. Phase I trial of welldifferentiated neuroendocrine tumors (NETs) with radiolabeled somatostatin antagonist ${ }^{177} \mathrm{Lu}$ satoreotide tetraxetan. Clin. Cancer Res. 25 6939-6947 (2019).

202. Jensen, R. T., Battey, J. F., Spindel, E. R. \& Benya, R. V. International Union of Pharmacology. LXVIII. Mammalian bombesin receptors: nomenclature, distribution, pharmacology, signaling, and functions in normal and disease states. Pharmacol. Rev. 60, 1-42 (2008).

203. Baratto, L., Jadvar, H. \& lagaru, A. Prostate cancer theranostics targeting gastrin-releasing peptide receptors. Mol. Imaging Biol. 20, 501-509 (2018).

204. Pooja, D., Gunukula, A., Gupta, N., Adams, D. J. \& Kulhari, H. Bombesin receptors as potential targets for anticancer drug delivery and imaging. Int. J. Biochem. Cell Biol. 114, 105567 (2019).

205. Morgat, C. et al. Comparison of the binding of the gastrin-releasing peptide receptor (GRP-R) antagonist ${ }^{68} \mathrm{Ga}-\mathrm{RM} 2$ and ${ }^{18} \mathrm{~F}$-FDG in breast cancer samples. PLOS ONE 14, e0210905 (2019).

206. Abouzayed, A. et al. Synthesis and preclinical evaluation of radio-iodinated GRPR/PSMA bispecific heterodimers for the theranostics application in prostate cancer. Pharmaceutics 11, 358 (2019).

207. Liolios, C., Sachpekidis, C., Schäfer, M. \& Kopka, K. Bispecific radioligands targeting prostate-specific membrane antigen and gastrin-releasing peptide receptors on the surface of prostate cancer cells. J. Label. Comp. Radiopharm. 62, 510-522 (2019).

208. Baratto, L., Duan, H., Maecke, H. R. \& lagaru, A. Imaging the distribution of gastrin releasing peptide receptors in cancer. J. Nucl. Med 61, 792-798 (2020)

209. Bodei, L. et al. Lu-177-AMBA bombesin analogue in hormone refractory prostate cancer patients: a phase escalation study with single-cycle administrations Eur. J. Nucl. Med. Mol. Imaging 34, S221 (2007).

210. Cescato, R. et al. Bombesin receptor antagonists may be preferable to agonists for tumor targeting. J. Nucl. Med. 49, 318-326 (2008). Comparative in vitro/in vivo study indicating that GRPR antagonists may be superior targeting agents to GRPR agonists

211. Dalm, S. U. et al. ${ }^{68} \mathrm{Ga} /{ }^{177} \mathrm{Lu}-\mathrm{NeoBOMB} 1$, a novel radiolabeled GRPR antagonist for theranostic use in oncology. J. Nucl. Med. 58, 293-299 (2017).

212. Kurth, J. et al. First-in-human dosimetry of gastrinreleasing peptide receptor antagonist $\left[{ }^{177} \mathrm{Lu}\right] \mathrm{Lu}-\mathrm{RM} 2$ : a radiopharmaceutical for the treatment of metastatic castration-resistant prostate cancer. Eur. J. Nucl. Med. Mol. Imaging 47, 123-135 (2020).

213. Thomas, V. A. \& Balthasar, J. P. Understanding inter-individual variability in monoclonal antibody disposition. Antibodies 8, E56 (2019).

214. Wohlrab, J. Pharmacokinetic characteristics of therapeutic antibodies. JDDG 13, 530-534 (2015)

215. Kohler, G. \& Milstein, C. Continuous cultures of fused cells secreting antibody of predefined specificity. Nature 256, 495-497 (1975).

Nobel Prize-winning work to produce antibodies from a single clone, an essential precursor to antibody-based RPT

216. Larson, S. M. et al. Imaging of melanoma with I-131-labeled monoclonal antibodies. J. Nucl. Med. 24, 123-129 (1983). Among the earliest reports of antibody-based cancer imaging; precursor to antibody-based RPT

217. Mach, J. P. et al. Tumor localization in patients by radiolabeled monoclonal antibodies against colon carcinoma. Cancer Res. 43, 5593-5600 (1983). Among the earliest reports of antibody-based cancer imaging; precursor to antibody-based RPT.

218. Colcher, D et al. Radiolabeled monoclonal-antibody B72. 3 localization in metastatic lesions of colorectal-cancer patients. Nucl. Med. Biol. 14 251 (1987).

219. Goldenberg, D. M. et al. Use of radiolabeled antibodies to carcinoembryonic antigen for the 
detection and localization of diverse cancers by external photoscanning. N. Engl. J. Med. 298 1384-1386 (1978).

\section{Among the earliest reports of antibody-based} cancer imaging; precursor to antibody-based RPT.

220. Mahe, M. A. et al. A phase II study of intraperitoneal radioimmunotherapy with iodine-131-labeled monoclonal antibody OC-125 in patients with residual ovarian carcinoma. Clin. Cancer Res. 5 (Suppl. 10), 3249-3253 (1999)

221. Cassaday, R. D. et al. Phase I study of a CD45targeted antibody-radionuclide conjugate for high-risk lymphoma. Clin. Cancer Res. 25, 6932-6938 (2019).

222. Tomlinson, B. K. et al. Rapid reduction of peripheral blasts in older patients with refractory acute myeloid leukemia (AML) using re-induction with single agent anti-CD45 targeted iodine (I-131) apamistamab lomab-B radioimmunotherapy in the phase III SIERRA trial. Clin. Lymphoma Myeloma Leukemia 19, S232 (2019).

223. Pagel, J. M. et al. Allogeneic hematopoietic cell transplantation after conditioning with I-131-antiCD45 antibody plus fludarabine and low-dose total body irradiation for elderly patients with advanced acute myeloid leukemia or high-risk myelodysplastic syndrome. Blood 114, 5444-5453 (2009).

224. Gopal, A. K., Pagel, J. M., Fromm, J. R., Wilbur, S \& Press, O. W. I-131 anti-CD45 radioimmunotherapy effectively targets and treats T-cell non-Hodgkin lymphoma. Blood 113, 5905-5910 (2009).

225. Mawad, R. et al. Radiolabeled anti-CD45 antibody with reduced-intensity conditioning and allogeneic transplantation for younger patients with advanced acute myeloid leukemia or myelodysplastic syndrome. Biol. Blood Marrow Transplant. 20, 1363-1368 (2014).

226. Tomlinson, B. et al Rapid reduction of peripheral blasts in older patients with refractory acute myeloid leukemia (AML) using reinduction with single agent anti-CD45 targeted iodine (I-131) apamistamab Iomab-B radioimmunotherapy in the phase III SIERRA trial. J. Clin. Oncol. 37 (Suppl. 15), 7048 (2019).

227. Griffeth, L. et al. Personalized dosimetry using 1-131-anti-CD45-apamistamab (lomab-B) prior to high-dose myeloablative radioimmunotherapy for hematopoietic stem cell transplant (HCT) in active, relapsed, or refractory acute myelogenous leukemia novel re-induction and targeted conditioning feasibility and engraftment results from the SIERRA trial. J. Nucl. Med. 60 (Suppl. 1), 434 (2019)

228. Pandit-Taskar, N. et al. Optimizing dosimetry imaging in high-dose radioimmunotherapy using the novel, anti-CD45 re-induction and targeted conditioning agent iodine (1-131) apamistamab lomab-B in patients 55 years or older with active, relapsed or refractory acute myeloid leukemia (SIE phase III trial). J. Nucl. Med. 60 (Suppl. 1), 433 (2019).

229. Schwartz, M. A. et al. Dose-escalation trial of M 195 labeled with I-131 for cytoreduction and marrow ablation in relapsed or refractory myeloid leukemias. J. Clin. Oncol. 11, 294-303 (1993).

230. Pagel, J. M. et al. Allogeneic hematopoietic cel transplantation after conditioning with ${ }^{131} \mathrm{I}$-anti-CD45 antibody plus fludarabine and low-dose total body irradiation for elderly patients with advanced acute myeloid leukemia or high-risk myelodysplastic syndrome. Blood 114, 5444-5453 (2009).

231. Press, O. W. et al. Phase II trial of ${ }^{131}{ }^{1}-\mathrm{B} 1$ (anti-CD20) antibody therapy with autologous stem cell transplantation for relapsed B cell lymphomas. Lancet 346, 336-340 (1995).

Use of RPT for bone marrow ablation in preparation for transplantation.

232. Orozco, J. J. et al. Anti-CD45 radioimmunotherapy using At-211 with bone marrow transplantation prolongs survival in a disseminated murine leukemia model. Blood 121, 3759-3767 (2013).

233. Li, Y. W. et al. cGMP production of astatine- 211 labeled anti-CD45 antibodies for use in allogeneic hematopoietic cell transplantation for treatment of advanced hematopoietic malignancies. Plos ONE 13, e0205135 (2018).

234. Scheinberg, D. A. et al. A phase I trial of monoclonal antibody M195 in acute myelogenous leukemia: specific bone marrow targeting and internalization of radionuclide. J. Clin. Oncol. 9, 478-490 (1991).

235. Sgouros, G. et al. Pharmacokinetics and dosimetry of an alpha-particle emitter labeled antibody:

${ }^{213} \mathrm{Bi}-\mathrm{HuM} 195$ (anti-CD33) in patients with leukemia. J. Nucl. Med. 40, 1935-1946 (1999). Dosimetry for a-emitter RPT in humans; one of the first such reports.
236. McDevitt, M. R., Finn, R. D., Sgouros, G., Ma, D. $\&$ Scheinberg, D. A. An ${ }^{225} \mathrm{Ac} /{ }^{213} \mathrm{Bi}$ generator system for therapeutic clinical applications: construction and operation. Appl. Radiat. Isot. 50, 895-904 (1999).

237. McDevitt, M. R., Finn, R. D., Ma, D., Larson, S. M. \& Scheinberg, D. A. Preparation of alpha-emitting 213Bi-labeled antibody constructs for clinical use. J. Nucl. Med. 40, 1722-1727 (1999).

238. Kolbert, K. S. et al. Parametric images of antibody pharmacokinetics in Bi213-HuM195 therapy of leukemia. J. Nucl. Med. 42, 27-32 (2001).

239. Rosenblat, T. L. et al. Sequential cytarabine and alphaparticle immunotherapy with bismuth-213-lintuzumab (HuM195) for acute myeloid leukemia. Clin. Cancer Res. 16, 5303-5311 (2010)

\section{Clinical $\alpha$-particle therapy.}

240. Jurcic, J. G. Targeted alpha-particle therapy for hematologic malignancies. J. Med. Imaging Radiat. Sci 50, S53-S57 (2019).

241. Jurcic, J. G. et al. Phase I trial of the targeted alpha-particle nano-generator actinium-225 ${ }^{225}$ Ac-lintuzumab) (anti-CD33; HuM 195) in acute myeloid leukemia (AML). J. Clin. Oncol. 29, 6516 (2011).

\section{Clinical $\alpha$-particle therapy}

242. Jurcic, J. G. et al. Phase I trial of the targeted alpha-particle nano-generator actinium-225 $\left({ }^{225} \mathrm{Ac}\right.$ )-lintuzumab (anti-CD33) in combination with low-dose cytarabine (LDAC) for older patients with untreated acute myeloid leukemia (AML). Blood 122, 1460 (2013).

243. Jurcic, J. G. Targeted alpha-particle therapy for hematologic malignancies. Semin. Nucl. Med. $\mathbf{5 0}$ 152-161 (2020)

244. Berger, M. S. et al. Actinium labeled daratumumab demonstrates enhanced killing of multiple myeloma cells over naked daratumumab. Blood 130, (2017).

245. Dadachova, E. et al. Ac-225-CD38 antibody targeting is effective and well tolerated in experimental models of lymphoma and multiple myeloma. J. Nucl. Med. 60 (Suppl. 1), 1410 (2019).

246. Juergens, R. A. et al. A phase I study of Ac-225-FPI1434 radioimmunotherapy in patients with IGF- $1 \mathrm{R}$ expressing solid tumors (Poster). J. Clin. Oncol. 37, TPS3152 (2019)

247. Hagemann, U. B. et al. Advances in precision oncology: targeted thorium-227 conjugates as a new modality in targeted alpha therapy. Cancer Biother. Radiopharm https://doi.org/10.1089/cbr.2020.3568 (2020).

248. Hagemann, U. B. et al. A novel high energy alpha pharmaceutical: in vitro and in vivo potency of a mesothelin-targeted thorium-227 conjugate (TTC) in a model of bone disease. Cancer Res. 76, 591 (2016)

249. Hagemann, U. B. et al. Mesothelin-targeted thorium-227 conjugate (MSLN-TTC): preclinical evaluation of a new targeted alpha therapy for mesothelin-positive cancers. Clin. Cancer Res. 25 4723-4734 (2019).

250. Hammer, S. et al. Preclinical pharmacology of the PSMA-targeted thorium-227 conjugate PSMA-TTC a novel targeted alpha therapeutic for the treatment of prostate cancer. Cancer Res. https://doi.org/ 10.1158/1538-7445.AM2017-5200 (2017).

251. Hammer, S. et al. Preclinical efficacy of a PSMAtargeted thorium-227 conjugate (PSMA-TTC), a targeted alpha therapy for prostate cancer Clin. Cancer Res. 26, 1985-1996 (2020).

252. Grant, D. et al. Pharmacokinetics and dosimetry of BAY 1862864, an alpha-emitting targeted thorium conjugate (CD22-TTC) in the Cynomolgus monkey. Eur. J. Nucl. Med. Mol. Imaging 45, S1 24 (2018)

253. Karlsson, J. et al. HER2-targeted thorium-227 conjugate (HER2-TTC): efficacy in a HER2 positive orthotopic bone model. Cancer Res. https://doi.org/ 10.1158/1538-7445.AM2017-5857 (2017).

254. Karlsson, J. et al. HER2-targeted thorium-227 conjugate (HER2-TTC): efficacy in preclinical models of trastuzumab and T-DM1 resistance. Cancer Res. https://doi.org/10.1158/1538-7445.AM2017-5859 (2017).

255. Karlsson, J. et al. In vitro and in vivo activity of a HER2-targeted thorium-227 conjugate (HER2-TTC) in HER2 low expressing and T-DM1/trastuzumab resistant preclinical mouse models. Eur. J. Cancer 103, E122 (2018).

256. Wickstroem, K. et al. Synergistic effect of a HER2 targeted thorium-227 conjugate in combination with olaparib in a BRCA2 deficient xenograft model. Pharmaceuticals 12, 155 (2019).

257. Waldmann, T. ABCs of radioisotopes used for radioimmunotherapy: alpha- and beta-emitters. Leuk. Lymphoma. 44, S107-S113 (2003).
258. Wahl, R. L. et al. lodine-131 anti-B1 antibody for B-cell lymphoma: an update on the Michigan phase I experience. J. Nucl. Med. 39 (Suppl. 8), 21-27 (1998).

259. Wahl, R. L. lodine-131 anti-B1 antibody therapy in non-Hodgkin's lymphoma: dosimetry and clinical implications. J. Nucl. Med. 39 (Suppl. 8), 1 (1998)

260. Wahl, R. L., Kroll, S. \& Zasadny, K. R. Patient-specific whole-body dosimetry: principles and a simplified method for clinical implementation. J. Nucl. Med. 39 (Suppl. 8), 14-20 (1998).

261. Witzig, T. E. et al. Treatment with ibritumomab tiuxetan radioimmunotherapy in patients with rituximab-refractory follicular non-Hodgkin's lymphoma. J. Clin. Oncol. 20, 3262-3269 (2002).

262. Davis, T. A. et al. Results of a randomized study of Bexxar (TM) (tositumomab and iodine I 131 tositumomab) vs. unlabeled tositumomab in patients with relapsed or refractory low-grade or transformed non-Hodgkin's lymphoma (NHL). Blood 98, 843A (2001).

263. Wahl, R. L. The clinical importance of dosimetry in radioimmunotherapy with tositumomab and iodine I 131 tositumomab. Semin. Oncol. 30, 31-38 (2003).

264. Baxter, L. T. \& Jain, R. K. Transport of fluid and macromolecules in tumors. IV. A microscopic model of the perivascular distribution. Microvasc. Res. 41 252-272 (1991).

265. Jain, R. K. \& Baxter, L. T. Mechanisms of heterogeneous distribution of monoclonal antibodies and other macromolecules in tumors: significance of elevated interstitial pressure. Cancer Res. 48 7022-7032 (1988).

266. Jain, R. K. Transport of molecules across tumor vasculature. Cancer Metastasis Rev. 6, 559-593 (1987).

267. Saga, T. et al. Targeting cancer micrometastases with monoclonal antibodies: a binding-site barrier. Proc. Natl Acad. Sci. USA 92, 8999-9003 (1995).

268. van Osdol, W., Fujimori, K. \& Weinstein, J. N. An analysis of monoclonal antibody distribution in microscopic tumor nodules: consequences of a "binding site barrier". Cancer Res. 51, 4776-4784 (1991).

269. Fujimori, K., Covell, D. G., Fletcher, J. E. \& Weinstein, J. N. A modeling analysis of monoclonal antibody percolation through tumors: a binding-site barrier. J. Nucl. Med. 31, 1191-1198 (1990).

270. Sgouros, G. Plasmapheresis in radioimmunotherapy of micrometastases: a mathematical modeling and dosimetrical analysis [see comments]. J. Nucl. Med. 33, 2167-2179 (1992)

271. Jaggi, J. S. et al. Improved tumor imaging and therapy via i.v. IgG-mediated time-sequential modulation of neonatal Fc receptor. J. Clin. Invest 117, 2422-2430 (2007).

272. Goodwin, D. A., Meares, C. F. \& Osen, M. Biological properties of biotin-chelate conjugates for pretargeted diagnosis and therapy with the avidin/biotin system. J. Nucl. Med. 39, 1813-1818 (1998). Initial approach to separating radionuclide delivery from cancer targeting to reduce haematological toxicity.

273. Verhoeven, M., Seimbille, Y. \& Dalm, S. U. Therapeutic applications of pretargeting. Pharmaceutics 11, 434 (2019).

274. Liapi, E. \& Geschwind, J.-F. H. Intra-arterial therapies for hepatocellular carcinoma: where do we stand? Ann. Surg. Oncol. 17, 1234-1246 (2010).

275. Lewandowski, R. J., Geschwind, J.-F., Liapi, E. \& Salem, R. Transcatheter intraarterial therapies: rationale and overview. Radiology 259, 641-657 (2011).

276. Lewandowski, R. J. \& Salem, R. Yttrium-90 radioembolization of hepatocellular carcinoma and metastatic disease to the liver. Semin. Intervent. Radiol. 23, 64-72 (2006)

277. Riaz, A., Awais, R. \& Salem, R. Side effects of yttrium-90 radioembolization. Front. Oncol. 4, 198 (2014).

278. Van Der Gucht, A. et al. Resin versus glass microspheres for ${ }^{90} \mathrm{Y}$ transarterial radioembolization: comparing survival in unresectable hepatocellular carcinoma using pretreatment partition model dosimetry. J. Nucl. Med. 58, 1334-1340 (2017).

279. Biederman, D. M. et al. Outcomes of radioembolization in the treatment of hepatocellular carcinoma with portal vein invasion: resin versus glass microspheres. J. Vasc. Intervent. Radiol. 27, 812-21 (2016).

280. Mumper, R. J., Ryo, U. Y. \& Jay, M. Neutronactivated holmium-166-poly (L-lactic acid) 
microspheres - a potential agent for the internal radiation-therapy of hepatic-tumors. J. Nucl. Med. 32 2139-2143 (1991)

281. Smits, M. L. J. et al. Holmium-166 radioembolisation in patients with unresectable, chemorefractory liver metastases (HEPAR trial): a phase 1, dose-escalation study. Lancet Oncol. 13, 1025-1034 (2012).

282. Arranja, A. G., Hennink, W. E., Chassagne, C. Denkova, A. G. \& Nijsen, J. F. W. Preparation and characterization of inorganic radioactive holmium-166 microspheres for internal radionuclide therapy. Mater. Sci. Eng. C Mater. Biol. Applications. 106, 110244 (2020).

283. Wang, X.-M. et al. Preventive effect of regional radiotherapy with phosphorus-32 glass microspheres in hepatocellular carcinoma recurrence after hepatectomy. World J. Gastroenterol. 14, 518-523 (2008).

284. Jeong, J. M. \& Chung, J.-K. Therapy with ${ }^{188}$ Re-labeled radiopharmaceuticals: an overview of promising results from initial clinical trials. Cancer Biother. Radiopharm. 18, 707-717 (2003).

285. Bretagne, J. F. et al. Hepatic artery injection of 1-131-labeled lipiodol. Part II. Preliminary results therapeutic use patients Hepatocellular carcinoma liver metastases. Radiology 168, 547-550 (1988).

286. Raoul, J. L. et al. Hepatic artery injection of I-131labeled lipiodol. Part I. Biodistribution study results in patients with hepatocellular carcinoma and liver metastases. Radiology 168, 541-545 (1988).

287. Raoul, J. I. et al. Internal radiation therapy for hepatocellular carcinoma. Results of a French multicenter phase II trial of transarterial injection of iodine 131-labeled Lipiodol. Cancer 69, 346-352 (1992).

288. Raoul, J. L. et al. Randomized controlled trial for hepatocellular carcinoma with portal vein thrombosis: intra-arterial iodine-131-iodized oil versus medical support. J. Nucl. Med. 35, 1782-1787 (1994).

289. Schwarz, L. et al. Adjuvant I-131 Lipiodol after resection or radiofrequency ablation for hepatocellular carcinoma. World J. Surg. 40, 1941-1950 (2016).

290. Lau, W. Y. et al. Adjuvant intra-arterial lipiodoliodine-131 for resectable hepatocellular carcinoma: a prospective randomised trial. Lancet 353, 797-801 (1999).

291. Lau, W. Y Lai, E. C. H. Leung, T. W. T \& Yu S. C. H. Adjuvant intra-arterial iodine-131-labeled lipiodol for resectable hepatocellular carcinoma: a prospective randomized trial-update on 5-year and 10-year survival. Ann. Surg. 247, 43-48 (2008).
292. Berenson, A. Market Forces Cited in Lymphoma Drugs' Disuse. New York Times https://www.nytimes. com/2007/07/14/health/1 4lymphoma.html (2007).

293. Mercadante, S. \& Fulfaro, F. Management of painfu bone metastases. Curr. Opin. Oncol. 19, 308-314 (2007).

294. John, K. U. S. DOE tri-lab production effort to provide accelerator-produced ${ }^{225} \mathrm{Ac}$ for radiotherapy: 2019 update. J. Nucl. Med. 60, 1612 (2019).

295. Hoehr, C. et al. Medical isotope production at TRIUMF - from imaging to treatment. Phys. Procedia. 90 200-208 (2017)

296. Karimian, A., Ji, N. T., Song, H. \& Sgouros, C. Mathematical modeling of pre-clinical alpha-emitter radiopharmaceutical therapy. Cancer Res. https://doi.org/10.1158/0008-5472.CAN-19-2553 (2019).

297. Eckerman, K. \& Endo, A. ICRP publication 107. Nuclear decay data for dosimetric calculations. Ann. ICRP 38, 7-96 (2007).

298. Papadimitroulas, P., Loudos, G., Nikiforidis, G. C. \& Kagadis, G. C. A dose point kernel database using GATE Monte Carlo simulation toolkit for nuclear medicine applications: comparison with other Monte Carlo codes. Med. Phys. 39, 5238-5247 (2012).

299. Simpkin, D. J. \& Mackie, T. R. EGS4 Monte-Carlo determination of the beta-dose kernel in water. Med. Phys. 17, 179-186 (1990).

300. [No authors listed.] Report 90: Key data for ionizing radiation dosimetry: measurement standards and applications. J. Int. Comm. Rad. Units Meas. https:// doi.org/10.1093/jicru/ndw043 (2016).

301. Schlom, J. et al. Monoclonal antibody-based therapy of a human tumor xenograft with a ${ }^{177}$ lutetium-labeled immunoconjugate. Cancer Res. 51, 2889-2896 (1991).

302. Kopka, K., Benešová, M., Barinka, C., Haberkorn, U. \& Babich, J. Glu-ureido-based inhibitors of prostatespecific membrane antigen: lessons learned during the development of a novel class of low-molecular-weight theranostic radiotracers. J. Nucl. Med. 58, 17S-26S (2017).

303. Maurer, T., Eiber, M., Schwaiger, M. \& Gschwend, J. E. Current use of PSMA-PET in prostate cancer management. Nat. Rev. Urol. 13, 226-235 (2016)

304. Muller, C. \& Schibli, R. Folic acid conjugates for nuclear imaging of folate receptor-positive cancer. J. Nucl. Med. 52, 1-4 (2011).

305. Larson, S. M., Carrasquillo, J. A., Cheung, N. K. \& Press, O. W. Radioimmunotherapy of human tumours. Nat. Rev. Cancer 15, 347-360 (2015).
306. Loevinger, R. \& Berman, M. A schema for calculating the absorbed dose from biologically distributed radionuclides. MIRD pamphlet no. 1. J. Nucl. Med. 9 , 5 (1968).

307. Ljungberg, M. et al. MIRD pamphlet no. 26: joint EANM/MIRD guidelines for quantitative ${ }^{177} \mathrm{Lu}$ SPECT applied for dosimetry of radiopharmaceutical therapy. J. Nucl. Med. 57, 151-162 (2016).

308. Prideaux, A. R. et al. Three-dimensional radiobiologic dosimetry: application of radiobiologic modeling to patient-specific 3-dimensional imaging-based internal dosimetry. J. Nucl. Med. 48, 1008-1016 (2007).

309. Sgouros, G. et al. Three-dimensional imaging-based radiobiological dosimetry. Semin. Nucl. Med. 38, 321-334 (2008).

310. Sgouros, G. et al. Treatment planning for internal radionuclide therapy: three-dimensional dosimetry for nonuniformly distributed radionuclides. J. NuCl. Med. 31, 1884-1891 (1990).

Imaging-based patient specific dosimetry for RPT

treatment planning.

Acknowledgements

G.S. acknowledges NIH grants R01CA116477 and R01CA187037.

\section{Competing interests}

G.S. is a founder of and holds equity in Radiopharmaceutical Imaging and Dosimetry LLC (Rapid). He serves as a member of Rapid's Board of Directors; this arrangement has been reviewed and approved by the Johns Hopkins University in accordance with its conflict of interest policies. M.M. was a consultant for Actinium Pharmaceuticals, Regeneron, Progenics, Bridge Medicine and General Electric. Memorial Sloan Kettering has filed for IP protection for inventions of M.M. related to alpha particle technology. L.B. was a non-paid consultant for Advanced Accelerator Applications (AAA), Ipsen, Clovis, ITM and Curium, and receives research support from AAA. Johns Hopkins University has filed for IP protection for inventions of J.N. related to alpha particle technology.

\section{Publisher's note}

Springer Nature remains neutral with regard to jurisdictional claims in published maps and institutional affiliations.

\section{Supplementary information}

Supplementary information is available for this paper at https://doi.org/10.1038/s41573-020-0073-9.

(C) Springer Nature Limited 2020 